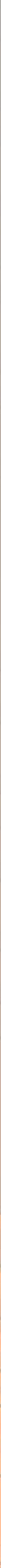




\title{
Vinberg's representations and arithmetic invariant theory
}

Jack A. Thorne

\begin{abstract}
Recently, Bhargava and others have proved very striking results about the average size of Selmer groups of Jacobians of algebraic curves over $\mathbb{Q}$ as these curves are varied through certain natural families. Their methods center around the idea of counting integral points in coregular representations, whose rational orbits can be shown to be related to Galois cohomology classes for the Jacobians of these algebraic curves.

In this paper we construct for each simply laced Dynkin diagram a coregular representation $(G, V)$ and a family of algebraic curves over the geometric quotient $V / / G$. We show that the arithmetic of the Jacobians of these curves is related to the arithmetic of the rational orbits of $G$. In the case of type $A_{2}$, we recover the correspondence between orbits and Galois cohomology classes used by Birch and Swinnerton-Dyer and later by Bhargava and Shankar in their works concerning the 2-Selmer groups of elliptic curves over $\mathbb{Q}$.
\end{abstract}

1. Introduction

2. Preliminaries: Vinberg theory, stable involutions, subregular elements

3. Subregular curves 2345

4. Jacobians and stabilizers of regular elements 2354

Acknowledgements 2366

References 2366

\section{Introduction}

This paper is a contribution to arithmetic invariant theory. Let $G$ be a reductive group over a field $k$, and let $V$ be a linear representation of $G$. Then the $\operatorname{ring} k[V]^{G}$ is a $k$-algebra of finite type, and we can define the quotient $V / / G=\operatorname{Spec} k[V]^{G}$ and a quotient map $\pi: V \rightarrow V / / G$. The determination of the structure of $k[V]^{G}$ and

This research was partially conducted during the period the author served as a Clay Research Fellow. MSC2010: primary 20G30; secondary 11E72.

Keywords: Arithmetic invariant theory, Galois cohomology, Arithmetic of algebraic curves. 
the fibers of $\pi$ falls under the rubric of geometric invariant theory, and is important in algebraic geometry.

In the case where $k$ is not algebraically closed, a further layer of difficulty is obtained by considering the $G(k)$-orbits in the fibers of $\pi$ over $k$-points of $V / / G$. This problem can be translated into the language of Galois cohomology, and as such often has close ties to arithmetic.

Bhargava has singled out those representations which are coregular, in the sense that $k[V]^{G}$ is isomorphic to a polynomial ring, as promising candidates for representations which may have interesting connections to arithmetic. For example, he has studied together with Shankar the case $G=\mathrm{SL}_{2}$ and $V=\mathrm{Sym}^{4} 2^{\vee}$, the space of binary quartic forms. In this case there are two independent polynomial invariants $I$ and $J$, and $k$-rational orbits with given values of $I$ and $J$ are related to classes in the Galois cohomology group $H^{1}(k, E[2])$ for the elliptic curve

$$
E: y^{2}=x^{3}+I x+J .
$$

These considerations have had very striking applications; see [Bhargava and Shankar 2010], or [Poonen 2013] for a beautiful summary. See also [Ho 2009] for a variety of similar orbit parametrizations associated to other representations, and [Bhargava and Ho 2013] for an exhaustive study of coregular representations related to genus-one curves. For each choice of pair $(G, V)$, one makes a construction in algebraic geometry which relates orbits in the given representation to algebraic curves, possibly with marked points, given line bundles, or other types of extra data.

By contrast, this paper represents a first effort to describe some of the phenomena appearing in arithmetic invariant theory through the lens of representation theory. We take as our starting point certain representations arising from Vinberg theory, whose role in arithmetic invariant theory has been emphasized by Gross. If $G$ is a reductive group over $k$ endowed with an automorphism $\theta$ of finite order $m$, then the fixed group $G^{\theta}$ acts on the $\theta=\zeta$ eigenspace $\mathfrak{g}_{1} \subset \mathfrak{g}=$ Lie $G$ for any choice $\zeta \in k^{\times}$of primitive $m$-th root of unity. Vinberg theory describes the geometric invariant theory of these representations. In the case when $\theta$ is regular and elliptic, in the sense of [Reeder et al. 2012], the generic element of $\mathfrak{g}_{1}$ will have a finite abelian stabilizer, and orbits in the representation are thus related to interesting Galois cohomology.

If $G$ is a split reductive group over $k$, then it has a unique $G^{\text {ad }}(k)$-conjugacy class of regular elliptic involutions $\theta$, characterized by the requirement that $\mathfrak{g}_{1}$ contain a regular nilpotent element. It is the representations associated to these canonical involutions for simple $G$ of type $A, D$ or $E$ that we study in this paper. We associate to each of these groups a family of algebraic curves, namely the smooth nearby fibers of a semiuniversal deformation of the corresponding simple plane curve 
singularity. The arithmetic of the Jacobians of these curves turns out to be related to the arithmetic of the rational orbits in the Vinberg representations. In each case the families of curves are universal families with marked points of fixed type. In types $A$ and $D$ we obtain families of hyperelliptic curves, while in types $E_{6}, E_{7}$ and $E_{8}$ we obtain families of nonhyperelliptic curves of genus respectively 3,3 and 4 .

Remark 1.1. In the forthcoming work [Bhargava and Ho 2013], the authors construct families of related coregular representations through the operations of symmetrization and skew-symmetrization. These representations are all related to the arithmetic of curves of genus one. For example, they consider the natural representation of $\mathrm{SL}_{2} \times \mathrm{SL}_{2} \times \mathrm{SL}_{2} \times \mathrm{SL}_{2}$ on $2 \otimes 2 \otimes 2 \otimes 2$, and its quadruple symmetrization yields the representation of $\mathrm{SL}_{2}$ on the space of binary quartic forms described above.

The quadruple skew-symmetrization, however, is not directly related to curves of genus one. In fact, the Vinberg representation we associate to $E_{7}$ is the representation of $\mathrm{SL}_{8} / \mu_{4}$ on $\wedge^{4} 8$. This is the quadruple skew-symmetrization of the above representation of $\mathrm{SL}_{2}^{4}$, and our work shows that its orbits are related to the arithmetic of the universal family of nonhyperelliptic curves of genus 3 with a rational flex in the canonical embedding.

Results. Let us now turn to a precise statement of our main results. For any unfamiliar notation relating to algebraic groups and their Lie algebras, we refer to the section on notation (page 2335). Let $k$ be a field of characteristic zero, and let $G$ be a split adjoint group over $k$ of type $A, D$ or $E$. We choose a regular elliptic involution $\theta$ of $G$ as described above, and set $G_{0}=\left(G^{\theta}\right)^{\circ}, \mathfrak{g}_{1}=\mathfrak{g}^{\theta=-1}$. Then $G_{0}$ acts on $\mathfrak{g}_{1}$ and a Chevalley-type restriction theorem holds for the pair $\left(G_{0}, \mathfrak{g}_{1}\right)$. In particular, the space $B=\mathfrak{g}_{1} / / G_{0}$ is isomorphic to affine $r$-space: $\mathfrak{g}_{1}$ is coregular. We write $\Delta \subset B$ for the discriminant divisor. Thus $\Delta$ is the image under $\pi: \mathfrak{g}_{1} \rightarrow B$ of the set of elements which are not regular semisimple.

Proposition 2.27 below implies that $\mathfrak{g}_{1}$ contains subregular nilpotent elements. We choose a subregular normal $\mathfrak{s l}_{2}$-triple $(e, h, f)$. (See Definition 2.16 for the definition of a normal $\mathfrak{s l}_{2}$-triple. To say that it is subregular simply means that $e$ and $f$ are subregular nilpotent elements of $\mathfrak{g}$.) Define $X=e+\mathfrak{z}_{\mathfrak{g}}(f)_{1}=e+\mathfrak{z}_{\mathfrak{g}}(f) \cap \mathfrak{g}_{1}$. Our first theorem concerns the natural map $X \hookrightarrow \mathfrak{g}_{1} \rightarrow B$.

Theorem 1.2. The morphism $X \rightarrow B$ is a flat family of reduced connected curves, smooth away from $\Delta$. The equations of these curves are given in the statement of Theorem 3.8.

In fact, $X$ is a transverse slice to the $G_{0}$-orbit of $e$ inside $\mathfrak{g}_{1}$, and $X \rightarrow B$ realizes a semiuniversal deformation of the central fiber $X_{0}$, which is an affine plane curve with a unique simple singularity of type equal to that of $G$. (For the definition of a simple curve singularity, we refer, for example, to [Cook 1998].) 
The following theorem incorporates results of Section 2 and Theorem 4.10.

Theorem 1.3. Let $x \in \mathfrak{g}_{1}$ be a regular semisimple element; equivalently, suppose that $b=\pi(x) \in B(k)$ does not lie inside $\Delta$.

- The stabilizer $Z_{G_{0}}(x)=Z_{b}$ is a finite abelian $k$-group and depends only on $b$ up to canonical isomorphism. This group is endowed with a nondegenerate alternating pairing $Z_{b} \times Z_{b} \rightarrow \mu_{2}$.

- Let $Y_{b}$ denote the smooth projective curve containing $X_{b}$ as a dense open subset. Let $J_{Y_{b}}$ denote its Jacobian variety. Then there is a canonical isomorphism of finite $k$-groups $J_{Y_{b}}[2] \cong Z_{b}$. Under this isomorphism the above pairing corresponds to the Weil pairing of $J_{Y_{b}}$.

Given $b \in(B \backslash \Delta)(k)$, we write $\mathfrak{g}_{1, b}=\pi^{-1}(b)$. If $K$ is a separable closure of $k$, then $\mathfrak{g}_{1, b}(K)$ consists of a single $G_{0}(K)$-orbit; the rational orbits in $\mathfrak{g}_{1, b}(k)$ are therefore classified by a suitable Galois cohomology set, with coefficients in $Z_{b} \cong J_{Y_{b}}$ [2]. The inclusion $X_{b} \subset \mathfrak{g}_{1, b}$ induces a map on rational points $X_{b}(k) \rightarrow \mathfrak{g}_{1, b}(k) / G_{0}(k)$. Our main theorem asserts that this map can in fact be interpreted in terms of 2-descent on the Jacobian $J_{Y_{b}}$ :

Theorem 1.4. There is a commutative diagram, functorial in $k$ :

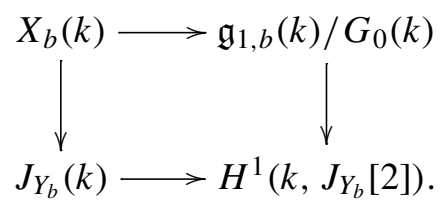

For the definitions of the arrows in this diagram, we refer to the statement of Theorem 4.15. If $G=\mathrm{PGL}_{3}$, then the family of curves $X \rightarrow B$ is the family $y^{2}=x^{3}+I x+J$ of genus-one curves described above, and we then recover the correspondence between orbits and Galois cohomology classes used by Bhargava and Shankar in their work on the average size of the 2-Selmer group of an elliptic curve over $\mathbb{Q}$. We are hopeful that the ideas discussed in this paper will have applications to the study of the average size of 2-Selmer groups beyond this case; compare the discussion following Conjecture 4.16.

Methods. Our methods are inspired primarily by work of Slodowy. Rational double point singularities of surfaces can be classified in terms of the Dynkin diagrams of simply laced simple algebraic groups. Grothendieck conjectured that one could give a representation-theoretic construction of this correspondence by looking at the generic singularity of the nilpotent cone of the corresponding group $G$. A proof of this conjecture was announced in a famous ICM lecture of Brieskorn [1971], but the first detailed proofs were given by Esnault [1980] and Slodowy [1980b]. Our work is what one obtains on combining the respective ideas of Slodowy and Vinberg. 
(After this work was completed, J. Sekiguchi informed us of some related earlier work [Sekiguchi and Shimizu 1981] where our families of curves also appear.)

Let us say a few words about the limits of our methods. Essential to our work is the use of $\mathfrak{s l}_{2}$-triples, whose existence relies in turn on the Jacobson-Morozov lemma. We must therefore work over a field of sufficiently large characteristic, relative to the Coxeter number of $G$. In this paper we choose for simplicity to work over a field of characteristic zero.

More serious is the lack of information we obtain about the image of the map $\mathfrak{g}_{1, b}(k) \rightarrow H^{1}\left(k, J_{Y_{b}}\right.$ [2]) constructed above. It follows from the above considerations that it contains the elements in the image under the 2-descent map $\delta: J_{Y_{b}}(k) \rightarrow H^{1}\left(k, J_{Y_{b}}[2]\right)$ of $X_{b}(k)$; we conjecture (page 2363) that it moreover contains the image under $\delta$ of the whole group $J_{Y_{b}}(k)$ of rational points of the Jacobian. In other words, we currently lack a way to construct sufficiently many orbits in the representations we study. We hope to return to this question in a future work.

Outline. Let us now outline the contents of this paper. In Section 2, we prove some basic properties of the so-called stable involutions $\theta$, and define the Vinberg representations to which they correspond. An important point here is the calculation of the stabilizers of the regular elements in $\mathfrak{g}_{1}$ in terms of the root datum of the ambient reductive group $G$. We also introduce the subregular nilpotent elements, and address the question of when $\mathfrak{g}_{1}$ contains subregular nilpotent elements which are defined over the base field $k$.

In Section 3, we construct the families of curves mentioned above inside a suitable transverse slice to the subregular nilpotent orbit.

Finally, in Section 4, we show how to relate the 2-torsion in the Jacobians of our curves and the stabilizers of regular elements, and prove our main theorem relating the 2-descent map to the classifying map for orbits in non-abelian Galois cohomology.

Other groups. In this paper we restrict to simple groups $G$ arising from simply laced Dynkin diagrams, and the corresponding Vinberg representations. One can try to apply our constructions to groups of nonsimply laced type. The families of curves thus obtained are versal deformations of planar curve singularities "with fixed symmetries"; this is the direct analogue for our context of the results in [Slodowy $1980 \mathrm{~b}, \S 6.2]$. The Jacobians of these curves admit a family of isogenies $\phi$, and it seems likely that some version of our main result continues to hold, with the groups $J_{Y_{b}}[\phi]$ now playing the role of the groups $J_{Y_{b}}[2]$.

Notation. As mentioned above, we work throughout over a field $k$ of characteristic zero. We assume basic familiarity with the theory of reductive groups over $k$, as 
studied, for example, in [Humphreys 1975] or [Springer 2009]. We assume that reductive groups are connected.

If $G$ is a reductive group acting linearly on a $k$-vector space $V$, then the ring of invariants $k[V]^{G}$ is a $k$-algebra of finite type (see, for example, [Springer 1977, Theorem 2.4.9]). We define $V / / G=\operatorname{Spec} k[V]^{G}$ and call it the categorical quotient. It in fact satisfies a universal property, but we will not need this here. We will write $\mathcal{N}(V)$ for the closed subscheme of $V$ cut out by the augmentation ideal of $k[V]^{G}$.

If $G, H, \ldots$ are algebraic groups then we will use gothic letters $\mathfrak{g}, \mathfrak{h}, \ldots$ to denote their Lie algebras. Let $G$ be a reductive group, and $T \subset G$ a split maximal torus. Then we shall write $\Phi_{\mathfrak{t}} \subset X^{*}(T)$ for the set of roots of $T$ in $\mathfrak{g}$, and $\Phi_{\mathfrak{t}}^{\vee} \subset X_{*}(T)$ for the set of coroots. The assignment $\alpha \in \Phi_{\mathfrak{t}} \mapsto d \alpha \in \mathfrak{t}^{*}$ identifies $\Phi_{\mathfrak{t}}$ with the set of roots of $\mathfrak{t}$ in $\mathfrak{g}$, and we will use this identification without comment. We write $W(\mathfrak{t})=N_{G}(T) / T$ for the Weyl group of $G$ with respect to $t$. We have the Cartan decomposition

$$
\mathfrak{g}=\mathfrak{t} \oplus \bigoplus_{\alpha \in \Phi_{\mathfrak{t}}} \mathfrak{g}^{\alpha}
$$

where $\operatorname{dim} \mathfrak{g}^{\alpha}=1$ for each $\alpha \in \Phi_{\mathfrak{t}}$. We write $U_{\alpha} \subset G$ for the unique $T$-invariant closed subgroup with Lie algebra $\mathfrak{g}_{\alpha}$ (see [Humphreys 1975, §26.3]). The tuple

$$
\left(X^{*}(T), \Phi_{\mathfrak{t}}, X_{*}(T), \Phi_{\mathfrak{t}}^{\vee}\right)
$$

is a root datum in the sense of [Springer 2009, §7.4]. We write $A_{G}$ for the center of $G$, and $\mathfrak{a}_{\mathfrak{g}}$ for its Lie algebra.

We will write $L_{G}=\mathbb{Z} \Phi_{\mathfrak{t}}$ for the root lattice of $G$ and $\Lambda_{G} \subset L_{G} \otimes_{\mathbb{Z}} \mathbb{Q}$ for the weight lattice of $L_{G}$. (These are the groups $Q$ and $P$, respectively, of [Bourbaki 1968, Chapter VI, §1.9].) If the group $G$ is clear from the context, we will omit the subscript $G$. We understand these to depend only on $G$ and not on $T$, so that $L_{G}$ and $\Lambda_{G}$ are defined up to (nonunique) isomorphism. We write $W_{G} \subset \operatorname{Aut}\left(L_{G}\right)$ for the corresponding Weyl group.

If $x \in \mathfrak{g}$, we write $Z_{G}(x)$ for its centralizer in $G$ under the adjoint representation, and $\mathfrak{z} \mathfrak{g}(x)$ for its centralizer in $\mathfrak{g}$. If $x$ is semisimple, then $Z_{G}(x)$ is reductive. Let $T \subset G$ be a maximal torus, and suppose that $x \in \mathfrak{t}$. Then $T \subset Z_{G}(x)$ is a maximal torus. Let

$$
\Phi_{\mathfrak{t}}(x)=\left\{\alpha \in \Phi_{\mathfrak{t}} \mid \alpha(x)=0\right\} \quad \text { and } \quad \Phi_{\mathfrak{t}}^{\vee}(x)=\left\{\alpha^{\vee} \in \Phi_{\mathfrak{t}}^{\vee} \mid \alpha \in \Phi_{\mathfrak{t}}(x)\right\} .
$$

Let $W(x)=Z_{W(\mathfrak{t})}(x)$. Then the root datum of $Z_{G}(x)$ is

$$
\left(X^{*}(T), \Phi_{\mathfrak{t}}(x), X_{*}(T), \Phi_{\mathfrak{t}}^{\vee}(x)\right),
$$

and the Weyl group of $Z_{G}(x)$ with respect to $T$ can be identified in a natural way with $W(x)$. 


\section{Preliminaries: Vinberg theory, stable involutions, subregular elements}

Throughout this section, $G$ is a split reductive group over a field $k$ of characteristic zero.

Elements of Vinberg theory. Let $\theta \in \operatorname{Aut}(G)$ be an automorphism of exact order $m>1$, and let $\zeta \in k$ be a primitive $m$-th root of unity. We will also write $\theta$ for the induced automorphism of $\mathfrak{g}$. We associate to $\theta$ the grading $\mathfrak{g}=\oplus_{i \in \mathbb{Z} / m \mathbb{Z}} \mathfrak{g}_{i}$, where by definition we have

$$
\mathfrak{g}_{i}=\left\{x \in \mathfrak{g} \mid \theta(x)=\zeta^{i} x\right\} .
$$

We write $G^{\theta}$ for the fixed subgroup of $\theta$, and $G_{0}$ for its connected component. Then Lie $G_{0}=\mathfrak{g}_{0}$, so the notation is consistent. The action of $G^{\theta}$ on $\mathfrak{g}$ leaves each $\mathfrak{g}_{i}$ invariant.

In what follows, we shall consider the representation of $G_{0}$ on the subspace $\mathfrak{g}_{1} \subset \mathfrak{g}$. The study of such representations is what we call Vinberg theory. For the basic facts about Vinberg theory, and in particular for proofs of the unproved assertions in this section, we refer to the papers [Vinberg 1976] or [Levy 2009].

Lemma 2.1. Let $x \in \mathfrak{g}_{1}$. Then $x$ can be written uniquely as $x=x_{s}+x_{n}$, where $x_{s}, x_{n}$ both lie in $\mathfrak{g}_{1}$ and are respectively semisimple and nilpotent.

Definition 2.2. A Cartan subspace $\mathfrak{c} \subset \mathfrak{g}_{1}$ is a maximal subalgebra consisting of semisimple elements. Note that $\mathfrak{c}$ is automatically abelian.

Proposition 2.3. Suppose that $k$ is algebraically closed. Then an element $x \in \mathfrak{g}_{1}$ is semisimple if and only if it is contained in a Cartan subspace, and all Cartan subspaces are $G_{0}(k)$-conjugate.

Let $\mathfrak{c} \subset \mathfrak{g}_{1}$ be a Cartan subspace, and define $W(\mathfrak{c}, \theta)=N_{G_{0}}(\mathfrak{c}) / Z_{G_{0}}(\mathfrak{c})$. This is the "little Weyl group" of the pair $(G, \theta)$. We define $\operatorname{rank} \theta=\operatorname{dim} \mathfrak{c}$. This is well-defined by Proposition 2.3.

The following result is contained in [Panyushev 2005, Theorem 1.1]. It is Vinberg's main result concerning the invariant theory of the representations considered here.

Theorem 2.4. 1. Restriction of functions induces an isomorphism

$$
k\left[\mathfrak{g}_{1}\right]^{G_{0}} \rightarrow k[\mathfrak{c}]^{W(\mathfrak{c}, \theta)} .
$$

Moreover, $W(\mathfrak{c}, \theta)$ is a (pseudo-)reflection group and $k[\mathfrak{c}]^{W(\mathfrak{c}, \theta)}$ is a polynomial ring in rank $\theta$ indeterminates.

2. Let $\pi: \mathfrak{g}_{1} \rightarrow \mathfrak{g}_{1} / / G_{0}$ denote the quotient map. Then $\pi$ is flat. If $k$ is algebraically closed, then for all $x \in \mathfrak{g}_{1}, \pi^{-1} \pi(x)$ consists of only finitely many $G_{0}(k)$-orbits. 
We say that $v \in \mathfrak{g}_{1}$ is stable if $G_{0} \cdot v$ is closed in $\mathfrak{g}_{1}$, and $Z_{G_{0}}(v)$ is finite. We say that $\theta$ is stable if $\mathfrak{g}_{1}$ contains stable elements. The property of being stable is hereditary, in the following sense.

Lemma 2.5. Suppose that $\theta$ is a stable automorphism. Let $x \in \mathfrak{g}_{1}$ be semisimple. Let $H=Z_{G}(x)$ and $\mathfrak{h}=$ Lie $H$. Then $\theta(H)=H$, and $\left.\theta\right|_{H}$ is a stable automorphism. Proof. Given $x$ as in the lemma, choose a Cartan subspace $\mathfrak{c}$ containing it. Then $\mathfrak{c}$ contains a stable vector, which is also stable when considered as an element of $\mathfrak{h}$; the result follows.

Stable involutions. In this paper we shall be particularly interested in the stable involutions.

Lemma 2.6. Suppose that $k$ is algebraically closed. There is a unique $G(k)$ conjugacy class of stable involutions $\theta$.

Proof. To show uniqueness, we reduce immediately to the case that $G$ is adjoint. By [Reeder et al. 2012, Lemma 5.6], any stable vector $v \in \mathfrak{g}_{1}$ is regular semisimple, and $\theta$ acts as -1 on its centralizer $\mathfrak{c}=\mathfrak{z}_{\mathfrak{g}}(v)$. In particular, we have $\mathfrak{c} \subset \mathfrak{g}_{1}$. It follows that the trace of $\theta$ on $\mathfrak{g}$ is equal to $-\operatorname{dim} \mathfrak{c}=-\operatorname{rank} G$, and a well-known theorem of E. Cartan asserts that this determines $\theta$ up to $G(k)$-conjugacy. We can also reduce existence to the case of $G$ adjoint. We will prove existence (even when $k$ is not algebraically closed) in this case below.

Lemma 2.7. Let $\theta$ be a stable involution of $G$. Then $\theta$ satisfies the following.

1. $\operatorname{rank} \theta=\operatorname{rank} G$.

2. There exists a maximal torus $C$ in $G$ on which $\theta$ acts by $x \mapsto x^{-1}$.

3. For all $x \in A_{G}$, we have $\theta(x)=x^{-1}$.

4. Let $\mathfrak{c}$ be a Cartan subspace (and hence, a Cartan subalgebra). Then the natural map $W(\mathfrak{c}, \theta) \rightarrow W(\mathfrak{c})$ is an isomorphism.

Proof. The first and second properties follow from the proof of Lemma 2.6. For the third property, we recall that $A_{G}$ is contained in any maximal torus of $G$. The final property is [Reeder et al. 2012, Corollary 7.4].

Suppose for the rest of this section that $\theta$ is a stable involution.

Proposition 2.8. Let $x=x_{s}+x_{n} \in \mathfrak{g}_{1}$ be a regular element. Then $Z_{G^{\theta}}(x)=$ $A_{Z_{G}\left(x_{s}\right)}$ [2]. In particular, this group is always finite and abelian.

Proof. We have $Z_{G}(x)=Z_{G}\left(x_{s}\right) \cap Z_{G}\left(x_{n}\right)$, so after replacing $G$ by $Z_{G}\left(x_{s}\right)$, we may assume that $x=x_{n}$ is a regular nilpotent element.

Then $Z_{G}(x)=A_{G} \cdot Z_{U}(x)$, a direct product, where $U$ is the unipotent radical of the unique Borel subgroup containing $x$. Quotienting by $A_{G}$, we may suppose that 
$G$ is adjoint and must show that $Z_{U}(x)^{\theta}$ is trivial. But since $x$ is regular, this is a finite unipotent group, so the result follows.

Corollary 2.9. Let $x=x_{s}+x_{n}$ be a regular element, and let $\mathfrak{c}$ be a Cartan subspace containing $x_{s}$. Let $C \subset G$ denote the maximal torus with Lie algebra $\mathfrak{c}$. Then

$$
Z_{G^{\theta}}(x) \cong \operatorname{Hom}\left(X^{*}(C) / 2 X^{*}(C)+\mathbb{Z} \Phi_{\mathfrak{c}}(x), \mathbb{G}_{m}\right) .
$$

Proof. For any reductive group $G$ with root datum $\left(X^{*}(T), \Phi_{\mathfrak{t}}, X_{*}(T), \Phi_{\mathfrak{t}}^{\vee}\right)$, there is a canonical isomorphism $X^{*}\left(A_{G}\right) \cong X^{*}(T) / \mathbb{Z} \Phi_{\mathfrak{t}}$. Now apply Proposition 2.8 .

Corollary 2.10. Suppose that $G$ is adjoint and that $k$ is algebraically closed. Let $x \in \mathfrak{g}_{1}$ be a regular semisimple element. Let L denote the root lattice of $G$, and $\Lambda \subset L \otimes_{\mathbb{Z}} \mathbb{Q}$ the weight lattice. Then there is an isomorphism

$$
Z_{G_{0}}(x) \cong \operatorname{Hom}\left(N, \mathbb{G}_{m}\right),
$$

well-defined up to conjugacy by the Weyl group $W$ of $L$, where $N$ denotes the image of $L$ in $\Lambda / 2 \Lambda$.

Proof. Let $G^{\text {sc }}$ denote the simply connected cover of $G$. Then $\theta$ acts on $G^{\text {sc }}$. A theorem of Steinberg - [Onishchik and Vinberg 1988, Chapter 4.4.8, Theorem 9] states that $\left(G^{\mathrm{sc}}\right)^{\theta}$ is connected, and hence $G_{0}$ is the image of the map $\left(G^{\mathrm{sc}}\right)^{\theta} \rightarrow G$. The present corollary now follows from the previous one.

Now suppose that the simple components of $G$ are simply laced (that is, their root systems are all of type $A, D$, or $E$ ), and let $L, \Lambda$ and $W$ be as in the statement of the corollary. Then there is a $W$-invariant quadratic form $\langle\cdot, \cdot\rangle: L \times L \rightarrow \mathbb{Z}$ uniquely determined by the requirement that $\langle\alpha, \alpha\rangle=2$ for every root $\alpha$. The pairing $\langle\cdot, \cdot\rangle$ on $L$ induces a pairing $(\cdot, \cdot): L / 2 L \times L / 2 L \rightarrow \mathbb{F}_{2}$. An easy calculation shows this pairing is alternating. In fact, we have the following:

Lemma 2.11. The pairing $(\cdot, \cdot)$ descends to a nondegenerate alternating pairing on $N$.

Proof. Suppose $x \in L$. Then the image of $x$ in $L / 2 L$ lies in the radical of $(\cdot, \cdot)$ if and only if $\langle x, L\rangle \subset 2 \mathbb{Z}$, if and only if $x \in 2 \Lambda$, since $\Lambda$ is the $\mathbb{Z}$-dual of $L$ with respect to the pairing $\langle\cdot, \cdot\rangle$.

Pairings of this type, associated to regular elliptic elements of Weyl groups, were first considered in [Reeder 2011].

Corollary 2.12. Suppose that $G$ is an adjoint group, and that the simple components of $G$ are simply laced. Then for any regular semisimple element $x \in \mathfrak{g}_{1}$, there is a canonical nondegenerate alternating form $(\cdot, \cdot): Z_{G_{0}}(x) \times Z_{G_{0}}(x) \rightarrow \mu_{2}$. 
We now show how to construct a stable involution over an arbitrary field $k$ of characteristic 0 . We let $G$ be a simple split adjoint group, and fix a split maximal torus $T$ and a Borel subgroup $B$ containing it. This determines a set $\Phi^{+} \subset \Phi=\Phi_{\mathrm{t}}$ of positive roots, and a root basis $R \subset \Phi^{+}$. We fix moreover for each $\alpha \in R$ a basis $X_{\alpha}$ of the one-dimensional vector space $\mathfrak{g}^{\alpha} \subset \mathfrak{g}$. The tuple $\left(T, B,\left\{X_{\alpha}\right\}_{\alpha \in R}\right)$ is called a pinning of $G$.

This choice of data determines a splitting $\operatorname{Aut}(G)=G \rtimes \Sigma$, where $\Sigma$ is the group of pinned automorphisms induced by automorphisms of the Dynkin diagram of $G$. On the other hand, writing $L=X^{*}(T)=\mathbb{Z} \Phi$ for the root lattice of $\mathfrak{g}$, the choice of root basis determines a splitting $\operatorname{Aut}(L)=W \rtimes \Sigma$ in a similar manner; see [Bourbaki 1975, Chapter VIII, §5.2]. We write $\sigma \in \Sigma$ for the image of $-1 \in \operatorname{Aut}(L)$, and define $\theta=\rho^{\vee}(-1) \rtimes \sigma \in \operatorname{Aut}(G)(k)$, where $\rho^{\vee} \in X_{*}(T)$ is the sum of the fundamental coweights.

Lemma 2.13. The automorphism $\theta$ is a stable involution.

Proof. This follows immediately from Corollary 5.7 of [Reeder et al. 2012].

This stable involution has good rationality properties. This is based on the following fact.

Lemma 2.14. Let $\theta$ be as above. Then $\mathfrak{g}_{1}$ contains a regular nilpotent element. Any two regular nilpotent elements of $\mathfrak{g}_{1}$ are conjugate by a unique element of $G^{\theta}(k)$.

Proof. The element $\sum_{\alpha \in R} X_{\alpha}$ is regular nilpotent and, by construction, lies in $\mathfrak{g}_{1}$. Fix a separable closure $K$ of $k$. If $E, E^{\prime} \in \mathfrak{g}_{1}$ are two regular nilpotent elements then they are conjugate by an element of $G^{\theta}(K)$. (This follows from [Levy 2007, Theorem 5.16].)

For any such $E$, the group $Z_{G^{\theta}}(E)$ is a finite unipotent group, and therefore trivial. It follows that $E, E^{\prime}$ are conjugate by a unique element of $G^{\theta}(K)$, which must therefore lie in $G^{\theta}(k)$.

Corollary 2.15. There is a unique $G(k)$-conjugacy class of stable involutions $\theta_{1}$ of $G$ such that there exists a regular nilpotent element $E_{1} \in \mathfrak{g}$ with $\theta_{1}\left(E_{1}\right)=-E_{1}$.

Proof. We have already proved the existence of such an element. For the uniqueness, fix again a separable closure $K$ of $k$. We have seen that $G(K)$ acts transitively on pairs $\left(\theta_{1}, E_{1}\right)$. On the other hand, the stabilizer of such a pair in $G(K)$ is trivial. It follows that any two such pairs are conjugate by a unique element of $G(k)$.

Definition 2.16. We call a tuple $(E, H, F)$ of elements of $\mathfrak{g}$ a normal $\mathfrak{s l}_{2}$-triple if it is an $\mathfrak{s l}_{2}$-triple, and moreover we have $E \in \mathfrak{g}_{1}, H \in \mathfrak{g}_{0}$, and $F \in \mathfrak{g}_{1}$.

Note that if $(E, H, F)$ is a normal $\mathfrak{s l}_{2}$-triple, then the restriction of $\theta$ to the subalgebra spanned by these elements is a stable involution.

Lemma 2.17. 1. Any nilpotent element $E \in \mathfrak{g}_{1}$ is contained in a normal $\mathfrak{s l}_{2}$-triple. 
2. Any two normal $\mathfrak{s l}_{2}$-triples $(E, H, F)$ and $\left(E, H^{\prime}, F^{\prime}\right)$ are $Z_{G_{0}}(E)(k)$-conjugate.

Proof. Fix a separable closure $K$ of $k$. For the first part, choose an arbitrary $\mathfrak{s l}_{2}-$ triple $(E, h, f)$ containing $E$, and decompose $h=h_{0}+h_{1}$ into $\theta$-eigenvectors. The argument of [Kostant and Rallis 1971, Proposition 4] implies that there is a unique $F \in \mathfrak{g}_{1} \otimes_{k} K$ such that $\left(E, h_{0}, F\right)$ is an $\mathfrak{s l}_{2}$-triple. But an $\mathfrak{s l}_{2}$-triple is determined uniquely by any 2 of its 3 elements, so descent implies that $F \in \mathfrak{g}_{1}$, and $\left(E, h_{0}, F\right)$ is the desired triple.

For the second part, we argue as in the proof of [Kostant and Rallis 1971, Proposition 4] and apply [Bourbaki 1975, Chapter VIII, §11.1, Lemma 4] to obtain the desired rationality property.

Corollary 2.18. The group $G(k)$ acts simply transitively on the set of pairs

$$
\left(\left(\theta_{1}\right),(E, H, F)\right)
$$

where $\theta_{1}$ is a stable involution of $G$ and $(E, H, F)$ is a normal $\mathfrak{s l}_{2}$-triple with respect to $\theta_{1}$ in which $E$ is a regular nilpotent element.

Example 2.19. We illustrate some of the concepts introduced so far in the case where $G$ is a split adjoint group of type $A_{2 r}$. Let $V$ be a vector space of dimension $2 r+1$, with basis $\left\{e_{1}, e_{2}, \ldots, e_{r}, v, f_{r}, \ldots, f_{2}, f_{1}\right\}$. We define an inner product $\langle\cdot, \cdot\rangle$ on $V$ by the formulae

$$
\left\langle e_{i}, e_{j}\right\rangle=0=\left\langle f_{i}, f_{j}\right\rangle=\left\langle e_{i}, v\right\rangle=\left\langle f_{i}, v\right\rangle
$$

for all $i, j$ and

$$
\langle v, v\rangle=1, \quad\left\langle e_{i}, f_{j}\right\rangle=\delta_{i j}
$$

If $T \in \operatorname{End}(V)$, write $T^{*}$ for the adjoint of $T$ with respect to this inner product. Then we take $G=\mathrm{PGL}_{2 \mathrm{r}+1}=\mathrm{PGL}(\mathrm{V})$, and $\theta: \mathfrak{s l}_{2 r+1} \rightarrow \mathfrak{s l}_{2 r+1}$ to be the involution $X \mapsto-X^{*}$. It is easy to check that $-\theta$ is just reflection in the antidiagonal. In particular, fixing the standard pinning $\left(T, B,\left\{X_{\alpha}\right\}_{\alpha \in R}\right)$ of $\mathfrak{s l}_{2 r+1}$, this $\theta$ is exactly the stable involution constructed of Lemma 2.13.

Then we see that $G^{\theta}=G_{0}=\mathrm{SO}(\mathrm{V})$ is connected, and we have

$$
\mathfrak{g}=\mathfrak{g}_{0} \oplus \mathfrak{g}_{1}, \quad \mathfrak{g}_{0}=\left\{X \in \operatorname{End}(V) \mid \operatorname{tr} X=0, X=-X^{*}\right\}=\mathfrak{s o}(V) .
$$

In particular, $\mathfrak{g}_{1}=\left\{X \in \operatorname{End}(V) \mid \operatorname{tr} X=0, X=X^{*}\right\}$ consists of the space of trace zero operators self-adjoint with respect to $\langle\cdot, \cdot\rangle$. 
The regular nilpotent element determined by the pinning is

$$
E=\left(\begin{array}{ccccc}
0 & 1 & 0 & \ldots & 0 \\
0 & 0 & 1 & 0 & \vdots \\
\vdots & \vdots & \vdots & \ddots & \vdots \\
0 & \ldots & 0 & 0 & 1 \\
0 & \ldots & 0 & 0 & 0
\end{array}\right) .
$$

These representations of odd orthogonal groups are exactly the ones used in [Bhargava and Gross 2013] to deal with the Selmer groups of hyperelliptic Jacobians.

Subregular elements. We recall that $x \in \mathfrak{g}$ is called subregular if $\operatorname{dim}_{\mathfrak{g}}(x)=$ rank $G+2$.

Proposition 2.20. The Lie algebra $\mathfrak{g}$ contains subregular nilpotent elements. Suppose that $G$ is simple and that $k$ is algebraically closed. Then there is a unique $G(k)$ orbit of subregular nilpotent elements in $\mathfrak{g}$, and these are dense in the complement of the regular nilpotent orbit in the nilpotent variety of $\mathfrak{g}$.

Proof. This follows from [Steinberg 1974, §3.10, Theorem 1].

Thus if $\mathfrak{g}$ is simple, then its nilpotent variety has a unique open orbit, consisting of regular nilpotent elements; its complement again has a unique open orbit, consisting of the subregular nilpotents. If $\mathfrak{g}=\mathfrak{g}_{1} \times \cdots \times \mathfrak{g}_{s}$ is a product of simple Lie algebras, then any nilpotent element $n$ can be written uniquely as a sum $n=n_{1}+\cdots+n_{s}$, where $n_{i} \in \mathfrak{g}_{i}$. It is then easy to see that $n$ is regular if and only if each $n_{i}$ is regular in $\mathfrak{g}$; and $n$ is subregular if and only if some $n_{i}$ is subregular in $\mathfrak{g}_{i}$, and all other $n_{j}$ are regular nilpotent elements. In particular, when $k$ is algebraically closed there are exactly $s G(k)$-orbits of subregular nilpotent elements, and there is a canonical bijection between these and the set of connected components of the Dynkin diagram of $\mathfrak{g}$.

Now suppose that $\theta$ is a stable involution of $G$. Before we continue, it is helpful to note the following.

Lemma 2.21. Let $x \in \mathfrak{g}_{1}$. Then

$$
\operatorname{dim} \mathfrak{z}_{\mathfrak{g}_{0}}(x)=\left(\operatorname{dim} \mathfrak{z}_{\mathfrak{g}}(x)-\operatorname{rank} G\right) / 2 \quad \text { and } \quad \operatorname{dim} G_{0} \cdot x=(\operatorname{dim} G \cdot x) / 2 .
$$

Proof. This follows from [Kostant and Rallis 1971, Proposition 5].

Our next goal is to show that $\mathfrak{g}_{1}$ contains subregular nilpotent elements. We use a trick based on the Kostant-Sekiguchi correspondence, which we now recall:

Theorem 2.22. Suppose that $k=\mathbb{R}$ and that $G$ is semisimple. Let $\tau$ be a Cartan involution of $G$. Then each of the following three sets is in canonical bijection with the others: 
1. The set of nilpotent $G(\mathbb{R})^{\circ}$-orbits in $\mathfrak{g}$.

2. The set of nilpotent $G^{\tau}(\mathbb{C})^{\circ}$-orbits in $\mathfrak{g}^{\tau=-1} \otimes_{\mathbb{R}} \mathbb{C}$.

3. The set of nilpotent $G_{0}(\mathbb{C})$-orbits in $\mathfrak{g}_{1} \otimes_{\mathbb{R}} \mathbb{C}$.

(Here we write $G(\mathbb{R})^{\circ}$ and $G^{\tau}(\mathbb{C})^{\circ}$ for the connected components of these groups in the analytic topology.) The map $G(\mathbb{R})^{\circ} \cdot X \mapsto G^{\tau}(\mathbb{C})^{\circ} \cdot X^{\prime}$ satisfies $G(\mathbb{C}) \cdot X=$ $G(\mathbb{C}) \cdot X^{\prime}$.

Proof. The bijection between the first two sets is constructed in [Collingwood and McGovern 1993, §9.5]. The existence of the bijection between the latter two follows since $\tau$ is a stable involution, and all such are conjugate over $\mathbb{C}$.

Corollary 2.23. Suppose that $k$ is algebraically closed. Then $\mathfrak{g}_{1}$ contains subregular nilpotent elements.

Proof. This is implied by Theorem 2.22 since, if $k=\mathbb{R}$ and $\mathfrak{g}$ is split, all conjugacy classes of nilpotent elements have an element defined over $k$.

To obtain more information, we must argue on a case-by-case basis. For the rest of this section, we assume that $G$ is adjoint, and that $\mathfrak{g}_{1}$ contains a regular nilpotent element. We first recall the following (see [Slodowy 1980b, §7.5, Lemma 4]).

Proposition 2.24. Suppose that $G$ is simple and simply laced, and let $x \in \mathfrak{g}$ be a subregular nilpotent element. Then $Z_{G}(x)$ is the semidirect product of a unipotent group with either $\mathbb{G}_{m}$ (if $G$ is type $A_{r}$ ) or the trivial group (if $G$ is of type $D_{r}$ or $E_{r}$ ). In particular, this centralizer is connected.

Corollary 2.25. Suppose that $k$ is algebraically closed, and that $G$ is of type $D_{r}$ or $E_{r}$. Then $\left(G^{\theta} / G_{0}\right)(k)$ acts simply transitively on the set of $G_{0}(k)$-orbits of subregular nilpotent elements of $\mathfrak{g}_{1}$.

Proof. Let $x$ be a subregular nilpotent element. Then $Z_{G^{\theta}}(x)=Z_{G_{0}}(x)$, by Proposition 2.24. It therefore suffices to show that $\#\left(G^{\theta} / G_{0}\right)(k)$ is equal to the number of real subregular nilpotent orbits. This can be accomplished, for example, by inspection of the tables in [Collingwood and McGovern 1993].

Proposition 2.26. Suppose that $k$ is algebraically closed, and that $G$ is of type $A_{r}$. Then there is a unique $G_{0}(k)$-conjugacy class of subregular nilpotent elements in $\mathfrak{g}_{1}$.

Proof. We note that when $k=\mathbb{R}$, there is a unique real orbit of subregular nilpotents in $\mathfrak{g}$.

We now treat the case where $k$ is not necessarily algebraically closed.

Proposition 2.27. The space $\mathfrak{g}_{1}$ contains a subregular nilpotent element. In particular, we can find normal $\mathfrak{s l}_{2}$-triples $(e, h, f)$ in $\mathfrak{g}$ with e a subregular nilpotent element. 
Proof. Let $K$ denote a separable closure of $k$. It suffices to find a normal $\mathfrak{s l}_{2}$-triple $(e, h, f)$ in $\mathfrak{g} \otimes_{k} K$ such that $e$ is subregular nilpotent and $h \in \mathfrak{g}$. For then the set of subregular elements is Zariski dense in $\mathfrak{g}_{1}^{\text {ad } h=2}$ (see [de Graaf 2011, Proposition 7]) and our chosen field $k$ is infinite.

Since $\mathfrak{g}_{1}$ contains a regular nilpotent element, we may assume that $G$ is equipped with a pinning ( $\left.T, B,\left\{X_{\alpha}\right\}_{\alpha \in R}\right)$ and that $\theta$ is the involution of Lemma 2.13, constructed in terms of this pinning. In particular, $\mathfrak{t}_{0}=\mathfrak{t}^{\theta} \subset \mathfrak{g}_{0}$ is a split Cartan subalgebra of $G_{0}$.

Let $(e, h, f)$ be a subregular normal $\mathfrak{s l}_{2}$-triple in $\mathfrak{g} \otimes_{k} K$. After conjugating by an element of $G_{0}(K)$, we can assume that $h$ lies in $\mathfrak{t}_{0} \otimes_{k} K \subset \mathfrak{t} \otimes_{k} K$. Now we have $\alpha(h) \in \mathbb{Z}$ for every root $\alpha$, since $h$ embeds in an $\mathfrak{s l}_{2}$-triple, and hence $h$ lies in $\mathfrak{t}_{0}$. The result follows.

Definition 2.28. We refer to a normal $\mathfrak{s l}_{2}$-triple $(e, h, f)$ with $e$ subregular as a subregular normal $\mathfrak{s l}_{2}$-triple.

Proposition 2.29. 1 . Suppose that $G$ is of type $D_{r}$ or $E_{r}$. Then all subregular nilpotent elements in $\mathfrak{g}_{1}$ are $G^{\theta}(k)$-conjugate.

2. Suppose that $G$ is of type $A_{2 r}$. Then there is a bijection between $k^{\times} /\left(k^{\times}\right)^{2}$ and the set of $G_{0}(k)$-orbits of subregular nilpotent elements in $\mathfrak{g}_{1}$, given by sending $d \cdot\left(k^{\times}\right)^{2}$ to the orbit of the element (in the notation of Example 2.19 above):

$$
\left(f_{1} \mapsto f_{2} \mapsto f_{3} \mapsto \cdots \mapsto f_{n} \mapsto d e_{n}, e_{n} \mapsto e_{n-1} \mapsto \cdots \mapsto e_{1}, v \mapsto 0\right) .
$$

3. Suppose that $G$ is of type $A_{2 r+1}$. Then all subregular nilpotent elements in $\mathfrak{g}_{1}$ are $G_{0}(k)$-conjugate.

Proof. Let $x \in \mathfrak{g}_{1}$ be a subregular nilpotent element. The first part follows since $Z_{G^{\theta}}(x)$ is a unipotent group, and hence has vanishing first Galois cohomology. To prove the second and third parts, we make an explicit calculation using the results of Kawanaka [1987]. Briefly, if $(e, h, f)$ is a normal $\mathfrak{s l}_{2}$-triple, let $\bar{G}_{0}$ denote the connected subgroup of $G$ with Lie algebra $\mathfrak{g}_{0} \cap \mathfrak{g}^{\text {ad } h=0}$. Then Kawanaka shows that $Z_{G_{0}}(e)$ has the form $C \ltimes R$, where $R$ is connected unipotent and $C=Z_{\bar{G}_{0}}(e)$ has reductive connected component. We summarize the results of this calculation here.

If $\mathfrak{g}$ is of type $A_{2 r}$, a choice of subregular nilpotent $x$ is the transformation given by the formula (in the notation of Example 2.19)

$$
f_{1} \mapsto f_{2} \mapsto f_{3} \mapsto \cdots \mapsto f_{n} \mapsto e_{n} \mapsto e_{n-1} \mapsto \cdots \mapsto e_{1}, v \mapsto 0 .
$$

If $d \in k^{\times}$, we define another element $x_{d}$ by the formula

$$
f_{1} \mapsto f_{2} \mapsto f_{3} \mapsto \cdots \mapsto f_{n} \mapsto d e_{n}, e_{n} \mapsto e_{n-1} \mapsto \cdots \mapsto e_{1}, v \mapsto 0 .
$$

One calculates that $Z_{G_{0}}(e)$ is a semidirect product of $\mu_{2}$ by a connected unipotent group, with Galois cohomology isomorphic (via the Kummer isomorphism) to 
$k^{\times} /\left(k^{\times}\right)^{2}$. With appropriate identifications the element $d \in k^{\times} /\left(k^{\times}\right)^{2}$ corresponds to the $G_{0}(k)$-orbit of the element $x_{d}$.

If $\mathfrak{g}$ is of type $A_{2 r+1}$, then one calculates that $Z_{G_{0}}(e)$ is connected unipotent, so has vanishing first Galois cohomology.

Proposition 2.30. Suppose that $k$ is algebraically closed. If $G$ is of type $A_{r}, D_{2 r+1}$ or $E_{r}$ then the closure of every regular nilpotent $G_{0}(k)$-orbit in $\mathfrak{g}_{1}$ contains every subregular nilpotent orbit.

If $G$ is of type $D_{2 r}$, then the closure of each regular nilpotent $G_{0}(k)$-orbit contains exactly 3 subregular nilpotent orbits, and each subregular nilpotent orbit is contained in the closure of exactly 3 regular nilpotent orbits.

Proof. The only cases needing proof are $A_{2 r+1}, D_{r}$, and $E_{7}$. The case of $A_{2 r+1}$ follows immediately, since $\left(G^{\theta} / G_{0}\right)(k)$ permutes the regular nilpotent orbits. The cases of $D_{r}$ and $E_{7}$ follow from the descriptions given in [Đoković and Litvinov 2003] and [Đoković 2001], respectively.

\section{Subregular curves}

For the rest of this paper, we fix the following notation. We suppose that $G$ is a split simple group over $k$, of type $A_{r}, D_{r}$, or $E_{r}$. We fix also a stable involution $\theta$ of $G$ and a regular nilpotent element $E \in \mathfrak{g}_{1}$. We recall that the pair $(\theta, E)$ is determined uniquely up to $G^{\text {ad }}(k)$-conjugacy. In this section we construct a family of curves over the categorical quotient $\mathfrak{g}_{1} / / G_{0}$. The construction is based on the notion of transverse slice to the action of an algebraic group, which we now briefly review.

Transverse slices. For the moment, let $H$ be an algebraic group acting on a variety $X$, both defined over $k$. Let $x \in X(k)$. By a transverse slice in $X$ to the orbit of $x$ (or more simply, a transverse slice at $x$ ), we mean a locally closed subvariety $S \subset X$ satisfying the following:

1. $x \in S(k)$.

2. The orbit map $H \times S \rightarrow X,(h, s) \mapsto h \cdot s$, is smooth.

3. $S$ has minimal dimension with respect to the above properties.

It is easy to show that if $X$ is smooth, then transverse slices of the above kind always exist and have dimension equal to the codimension of the orbit $H \cdot x$ in $X$. (Here we use that $k$ is of characteristic zero; in general, one should assume also that the orbit maps are separable.) An important property of transverse slices is the following slight extension of [Slodowy 1980b, §5.2, Lemma 3]:

Proposition 3.1. Let $H$ be an algebraic group acting on a smooth variety $X$. Let $S_{1}, S_{2}$ be transverse slices at points $x_{1}, x_{2} \in X(k)$, respectively, where $x_{1}, x_{2}$ lie in 
the same $H(k)$-orbit of $X$. Let $f: X \rightarrow Y$ be an $H$-equivariant morphism, where $H$ acts trivially on $Y$.

- $S_{1}, S_{2}$ are étale locally isomorphic over $Y$ in the sense that there exists a variety $S$ over $Y$ with a geometric point $\bar{s}$ and étale $Y$-morphisms $\phi_{1}: S \rightarrow S_{1}$, $\phi_{2}: S \rightarrow S_{2}$ with $\phi_{1}(\bar{s})=x_{1}, \phi_{2}(\bar{s})=x_{2}$.

- Suppose further that $k=\mathbb{C}$. Then $S_{1}(\mathbb{C}), S_{2}(\mathbb{C})$ are locally isomorphic over $Y(\mathbb{C})$ in the analytic topology. Furthermore, there exist arbitrarily small neighborhoods $U_{1} \subset S_{1}(\mathbb{C}), U_{2} \subset S_{2}(\mathbb{C})$ of $x$ and analytic isomorphisms $\psi: U_{1} \rightarrow U_{2}$ over $Y(\mathbb{C})$ such that the induced maps

$$
U_{1} \hookrightarrow X(\mathbb{C}), \quad U_{1} \cong U_{2} \hookrightarrow X(\mathbb{C})
$$

are homotopic over $Y(\mathbb{C})$.

An important special case where we can construct transverse slices explicitly is the case of a reductive group $H$ acting via the adjoint representation on its Lie algebra $\mathfrak{h}$. Let $(e, h, f)$ be an $\mathfrak{s l}_{2}$-triple in $\mathfrak{h}$.

Proposition 3.2. $S=e+\mathfrak{z}_{\mathfrak{h}}(f)$ is a transverse slice to the action of $H$ at every point of $S$. In other words, the multiplication map $\mu: H \times S \rightarrow \mathfrak{h}$ is everywhere smooth.

The proof is based on the following construction of Slodowy. First, we may decompose $\mathfrak{h}=\oplus_{i} V_{i}$ into a direct sum of irreducible submodules under the adjoint action of the $\mathfrak{s l}_{2}$ spanned by $e, h$, and $f$. We let $\lambda: \mathbb{G}_{m} \rightarrow H$ be the cocharacter with $d \lambda(1)=h$. Let $p_{1}, \ldots, p_{r}$ be algebraically independent homogeneous polynomials generating the ring of invariants $k[\mathfrak{h}]^{H}$. (We remind the reader that the adjoint representation of $H$ on $\mathfrak{h}$ is coregular, so such elements certainly exist.) We suppose that they have degrees $d_{1}, \ldots, d_{r}$. We suppose that $V_{i}$ has dimension $m_{i}$, and choose for each $i$ a basis vector $v_{i}$ of the lowest weight space of $V_{i}$.

A general element $v \in S$ can be written in the form $v=e+\sum_{i} x_{i} v_{i}$, and we have

$$
\lambda(t)(v)=t^{2} e+\sum_{i} t^{1-m_{i}} x_{i} v_{i}, \quad t v=t e+\sum_{i} t x_{i} v_{i}
$$

and

$$
p_{i}(\lambda(t)(v))=p_{i}(v), \quad p_{i}(t v)=t^{d_{i}} p_{i}(v) .
$$

Defining an action $\rho$ of $\mathbb{G}_{m}$ on $\mathfrak{h}$ by $\rho(t)(v)=t^{2} \lambda\left(t^{-1}\right)(v)$, we see that $S$ is $\rho$ invariant, and the $\rho$-action contracts $S$ to $e$. If we let $\mathbb{G}_{m}$ act on $\mathfrak{h} / / H$ by the square of its usual action, then the composite $S \hookrightarrow \mathfrak{h} \rightarrow \mathfrak{h} / / H$ becomes $\mathbb{G}_{m}$-equivariant. In other words, writing $w_{1}, \ldots, w_{n}$ for the weights of the $\rho$-action on $S$, the morphism $S \rightarrow \mathfrak{h} / / H$ is quasihomogeneous of type $\left(d_{1}, \ldots, d_{r} ; w_{1}, \ldots, w_{n}\right)$. The weights $w_{i}$ are given by the formula $w_{i}=m_{i}+1$. 
Proof of Proposition 3.2. Define an action of $\mathbb{G}_{m} \times H$ on $H \times S$ by

$$
(t, g) \cdot(k, s)=(g k \lambda(t), \rho(t)(s)),
$$

and let $\mathbb{G}_{m} \times H$ act on $\mathfrak{h}$ by $(t, g) \cdot X=t^{2} g(X)$. Then the map $\mu: H \times S \rightarrow \mathfrak{h}$ is equivariant for these actions, and smooth in a neighborhood of $H \times\{e\} \subset H \times S$; since the $\mathbb{G}_{m}$-actions are contracting, it follows that $\mu$ is smooth everywhere.

Corollary 3.3. The composite $S \hookrightarrow \mathfrak{h} \rightarrow \mathfrak{h} / / H$ is faithfully flat.

Proof. The composite $H \times S \rightarrow S \rightarrow \mathfrak{h} / / H$ is equal to the composite

$$
H \times S \rightarrow \mathfrak{h} \rightarrow \mathfrak{h} / / H,
$$

which is a composition of flat morphisms, hence flat $(H \times S \rightarrow \mathfrak{h}$ is flat since we have just proved it to be smooth). Since the second projection $H \times S \rightarrow S$ is flat, $S \rightarrow \mathfrak{h} / / H$ must also be flat.

The image is a $\mathbb{G}_{m}$-stable open subset of $\mathfrak{h} / / H$ containing the origin, hence the whole of $\mathfrak{h} / / H$. The faithful flatness follows.

Let us now return to our group $G$ with stable involution $\theta$, and let $(e, h, f)$ now denote a normal $\mathfrak{s l}_{2}$-triple. From the above, we see that there is a direct sum decomposition $\mathfrak{g}=[e, \mathfrak{g}] \oplus \mathfrak{z}_{\mathfrak{g}}(f)$. Both summands are $\theta$-stable so we deduce that $\mathfrak{g}_{1}=\left[e, \mathfrak{g}_{0}\right] \oplus \mathfrak{z}_{\mathfrak{g}}(f)_{1}$, where by definition $\mathfrak{z}_{\mathfrak{g}}(f)_{1}=\mathfrak{z}_{\mathfrak{g}}(f) \cap \mathfrak{g}_{1}$. It follows that $X=e+\mathfrak{z}_{\mathfrak{g}}(f)_{1}$ is a transverse slice at $e \in \mathfrak{g}_{1}$, and the contracting $\mathbb{G}_{m}$-action on $e+\mathfrak{z}_{\mathfrak{g}}(f)$ leaves $X$ invariant. Identical arguments to those above now prove the following.

Proposition 3.4. The map $\mu: G_{0} \times X \rightarrow \mathfrak{g}_{1}$ is smooth and the composite $X \hookrightarrow \mathfrak{g}_{1} \rightarrow$ $\mathfrak{g}_{1} / / G_{0}$ is faithfully flat.

We now examine two special cases of this construction in more detail.

The regular $\mathfrak{s l}_{2}$ and the Kostant section. Let $d_{1}, \ldots, d_{r}$ denote the degrees of algebraically independent homogeneous generators of the polynomial ring $k\left[\mathfrak{g}_{1}\right]^{G_{0}}$. We let $(E, H, F)$ be the unique normal $\mathfrak{s l}_{2}$-triple containing the element $E$, and set $\kappa=E+\mathfrak{z}_{\mathfrak{g}}(F)_{1}$. We call $\kappa$ the Kostant section. It has the following remarkable properties.

Lemma 3.5. The composite $\kappa \hookrightarrow \mathfrak{g}_{1} \rightarrow \mathfrak{g}_{1} / / G_{0}$ is an isomorphism. Every element of $\kappa$ is regular. In particular, the map $\mathfrak{g}_{1}(k) \rightarrow\left(\mathfrak{g}_{1} / / G_{0}\right)(k)$ is surjective, and if $k$ is algebraically closed then $\kappa$ meets every $G_{0}(k)$-conjugacy class of regular semisimple elements.

Proof. It is well-known that in this case the map $\kappa \rightarrow \mathfrak{g}_{1} / / G_{0}$ is quasihomogeneous of type $\left(2 d_{1}, \ldots, 2 d_{r} ; 2 d_{1}, \ldots, 2 d_{r}\right)$; compare [Panyushev 2005, proof of Theorem 3.3]. Lemma 3.14 now implies that it must be an isomorphism. The remaining claims are immediate. 
A subregular $\mathfrak{s l}_{2}$. Fix a normal subregular $\mathfrak{s l}_{2}$-triple $(e, h, f)$, and set $X=e+\mathfrak{z}_{\mathfrak{g}}(f)_{1}$. (Note that if $G$ is of type $A_{1}$, then there is no nonzero subregular nilpotent element, and therefore no subregular $\mathfrak{s l}_{2}$-triple, since by definition an $\mathfrak{s l}_{2}$-triple consists of 3 linearly independent elements. In this case, we just take $X=\mathfrak{g}_{1}$.) Recall that we have defined an action of $\mathbb{G}_{m}$ on $X$.

Proposition 3.6. We have $\operatorname{dim} X=r+1$. We write $w_{1}, \ldots, w_{r+1}$ for the weights of the $\mathbb{G}_{m}$-action. After reordering, we have $w_{i}=2 d_{i}$ for $i=1, \ldots, r-1$. The $2 d_{i}, i=1, \ldots, r-1$ and $w_{r}$ and $w_{r+1}$ are given in the following table:

\begin{tabular}{c|ccccccc|c|cc}
\hline & $2 d_{1}$ & $2 d_{2}$ & $2 d_{3}$ & $\cdots$ & $\cdots$ & $2 d_{r-2}$ & $2 d_{r-1}$ & $2 d_{r}$ & $w_{r}$ & $w_{r+1}$ \\
\hline$A_{r}$ & 4 & 6 & 8 & $\cdots$ & $\cdots$ & $2 r-2$ & $2 r$ & $2 r+2$ & 2 & $r+1$ \\
$D_{r}$ & 4 & 8 & 12 & $\cdots$ & $\cdots$ & $4 r-8$ & $2 r$ & $4 r-4$ & 4 & $2 r-4$ \\
$E_{6}$ & 4 & 10 & 12 & & & 16 & 18 & 24 & 6 & 8 \\
$E_{7}$ & 4 & 12 & 16 & & 20 & 24 & 28 & 36 & 8 & 12 \\
$E_{8}$ & 4 & 16 & 24 & 28 & 36 & 40 & 48 & 60 & 12 & 20 \\
\hline
\end{tabular}

Proof. The proof is by explicit calculation, along similar lines to the proof of [Slodowy 1980b, §7.4, Proposition 2]. We describe the method. If $V \subset \mathfrak{g}$ is a $\theta$-stable simple $\mathfrak{s l}_{2}$-submodule, then its highest weight space is $\theta$-invariant. Moreover, the eigenvalue of $\theta$ on this highest weight space determines the action of $\theta$ on every weight space. We can calculate a decomposition of $\mathfrak{g}$ into a direct sum of $\theta$-stable simple $\mathfrak{s l}_{2}$-modules by calculating the dimension of each weight space of $h$, and the trace of $\theta$ on each weight space. This can be accomplished by using the explicit $\theta$ constructed in Lemma 2.13 and a list of the roots of $\mathfrak{g}$. We can then fill in the table by reading off the lowest weight spaces which have $\theta$-eigenvalue equal to -1 .

Example 3.7. We illustrate the method of proof in the case that $G$ is of type $A_{2}$. Then a choice of $h$ is

$$
h=\left(\begin{array}{rrr}
1 & 0 & 0 \\
0 & 0 & 0 \\
0 & 0 & -1
\end{array}\right),
$$

in the notation of Example 2.19. We can write the weights of $h$ on $\mathfrak{g}$ with multiplicity as follows:

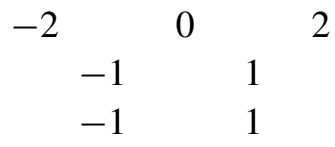

Thus $\mathfrak{g}$ decomposes as a direct sum $V(3) \oplus V(2) \oplus V(2) \oplus V(1)$, where $V(i)$ denotes the unique isomorphism class of $\mathfrak{s l}_{2}$-modules of dimension $i$. In this case -1 is an eigenvalue of $\theta$ of multiplicity 1 on each weight space. (Recall that $-\theta$ is 
reflection in the antidiagonal.) We can now decorate each weight space with a + or - , according to its $\theta$-eigenvalue:

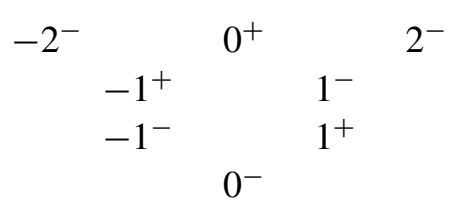

It follows that $\operatorname{dim}_{\mathfrak{z} \mathfrak{g}}(f)_{1}=3$, as expected, and the eigenvalues of $h$ on $\mathfrak{z}_{\mathfrak{g}}(f)_{1}$ are $-2,-1$ and 0 , hence the weights on $e+\mathfrak{z}_{\mathfrak{g}}(f)_{1}$ are 2,3 and 4 .

Henceforth we write $\mathfrak{g}_{1} / / G_{0}=B$, and $\varphi: X \rightarrow B$ for the restriction of the quotient map $\pi: \mathfrak{g}_{1} \rightarrow \mathfrak{g}_{1} / / G_{0}$ to $X$. The main result of this section is the following.

Theorem 3.8. The fibers of $\varphi$ are reduced curves. The central fiber $X_{0}=\varphi^{-1}(0)$ has a unique singular point which is a simple singularity of type $A_{r}, D_{r}, E_{r}$, corresponding to that of $G$. We can choose homogeneous coordinates $\left(p_{d_{1}}, \ldots, p_{d_{r}}\right)$ on $B$ and $\left(p_{d_{1}}, \ldots, p_{d_{r-1}}, x, y\right)$ on $X$ such that the family $X \rightarrow B$ of curves is as given by the following table:

\begin{tabular}{l|l}
\hline$G$ & $X$ \\
\hline$A_{r}$ & $y^{2}=x^{r+1}+p_{2} x^{r-1}+\cdots+p_{r+1}$ \\
$D_{r}$ & $y\left(x y+p_{r}\right)=x^{r-1}+p_{2} x^{r-2}+\cdots+p_{2 r-2}$ \\
$E_{6}$ & $y^{3}=x^{4}+y\left(p_{2} x^{2}+p_{5} x+p_{8}\right)+p_{6} x^{2}+p_{9} x+p_{12}$ \\
$E_{7}$ & $y^{3}=x^{3} y+p_{10} x^{2}+x\left(p_{2} y^{2}+p_{8} y^{2}+p_{14}\right)+p_{6} y^{2}+p_{12} y+p_{18}$ \\
$E_{8}$ & $y^{3}=x^{5}+y\left(p_{2} x^{3}+p_{8} x^{2}+p_{14} x+p_{20}\right)+p_{12} x^{3}+p_{18} x^{2}+p_{24} x+p_{30}$ \\
\hline
\end{tabular}

(This means, for example, that when $G$ is of type $A_{r}$, the relation $p_{r+1}=$ $y^{2}-\left(x^{r+1}+p_{2} x^{r-1}+\cdots+p_{r} x\right)$ holds on $X$.) The proof of Theorem 3.8 follows closely the one in [Slodowy 1980b], with some simplifications due to the fact that we work with curves, rather than surfaces. We begin with some general considerations, and reduce to a case-by-case calculation using the invariant degrees of $G$.

The possibility of choosing coordinates as above is a consequence of the next result.

Lemma 3.9 [Slodowy 1980b, §8.1, Lemma 2]. Let $V$ and $U$ be $k$-vector spaces of dimensions $m$ and $n$, respectively, on which $\mathbb{G}_{m}$ acts linearly. Let $\phi: V \rightarrow U$ be a morphism equivariant for these actions. Suppose that $d \phi_{0}$ has rank $s$ and that $\mathbb{G}_{m}$ acts with strictly positive weights on $U$ and $V$.

Then there exist $\mathbb{G}_{m}$-invariant decompositions $V=V_{0} \oplus W, U=U_{0} \oplus W$, $\operatorname{dim} W=s$, and a regular automorphism $\alpha$ of $V$ such that $\phi \circ \alpha$ has the form $\left(v_{0}, w\right) \mapsto\left(\psi\left(v_{0}, w\right), w\right)$ for some morphism $\psi: V_{0} \oplus W \rightarrow U_{0}$.

To apply this to the map $\varphi: X \rightarrow B$, we need the following result. 
Proposition 3.10. Let $x \in X$. Then $d \varphi_{x}$ has maximal rank $r=\operatorname{rank} G$ if and only if $x$ is a regular element. The map $d \varphi_{0}: T_{e} X \rightarrow T_{0} B$ has rank $r-1$.

Proof. Let $p: \mathfrak{g} \rightarrow \mathfrak{g} / / G$ denote the adjoint quotient map. For any $y \in \mathfrak{g}_{1}$, we have $d p_{y}\left(\mathfrak{g}_{0}\right)=0$. This is true if $y$ is regular, since then $\mathfrak{g}_{0}=\left[y, \mathfrak{g}_{1}\right] \subset[y, \mathfrak{g}]$ is contained in the tangent space to the orbit $G \cdot y$. It then follows for any $y \in \mathfrak{g}_{1}$, since the regular elements are dense. In particular, we have rank $d p_{y}=\operatorname{rank} d \pi_{y}=\operatorname{rank} d \varphi_{y}$ for any $y \in X$. The first part of the proposition now follows, since $y \in \mathfrak{g}_{1}$ is regular if and only if $d p_{y}$ has maximal rank.

For the second part, we remark that rank $d p_{e}=r-1$ if $e$ is subregular nilpotent, by [Slodowy 1980b, §8.3, Proposition 1].

We thus obtain a decomposition of affine spaces $X=V_{0} \oplus W, B=U_{0} \oplus W$, where $\operatorname{dim} W=r-1, \operatorname{dim} V_{0}=2$, and $\operatorname{dim} U_{0}=1$. With respect to these decompositions we write $\varphi: V_{0} \oplus W \rightarrow U_{0} \oplus W$ in the form $\varphi\left(v_{0}, w\right)=\left(\psi\left(v_{0}, w\right), w\right)$.

Recall that $\varphi$ is $\mathbb{G}_{m}$-equivariant of type $\left(2 d_{1}, \ldots, 2 d_{r} ; w_{1}, \ldots, w_{r+1}\right)$. By inspection of the tables above, we have $2 d_{r}>w_{i}$, each $i=1, \ldots, r+1$, and hence the weights occurring in $W$ are $2 d_{1}, \ldots, 2 d_{r-1}$. Moreover, the unique weight of $U_{0}$ is given by $2 d_{r}$ and the weights of $V_{0}$ are $w_{r}, w_{r+1}$. Let $x, y$ be homogeneous coordinates on $V_{0}$ of weight $w_{r}$ and $w_{r+1}$, respectively. It follows that $X_{0} \subset V_{0}$ is cut out by a quasihomogeneous polynomial $f(x, y)$ of type $\left(2 d_{r} ; w_{r}, w_{r+1}\right)$.

Proposition 3.11. After possibly making a linear change of variables, the polynomial $f(x, y)$ is as given by the following table.

\begin{tabular}{cc}
\hline$G$ & $f(x, y)$ \\
\hline$A_{r}, r \geq 1$ & $y^{2}-x^{r+1}$ \\
$D_{r}, r \geq 4$ & $x y^{2}-x^{r-1}$ \\
$E_{6}$ & $y^{3}-x^{4}$ \\
$E_{7}$ & $y^{3}-x^{3} y$ \\
$E_{8}$ & $y^{3}-x^{5}$ \\
\hline
\end{tabular}

Proof. We suppose first that $k$ is algebraically closed. Then the induced map $G_{0} \times X_{0} \rightarrow \pi^{-1}(0)$ is smooth, since $X$ is a transverse slice and this property is preserved under passage to fibers (see [Slodowy 1980b, §5, Lemma 2]). Since $\pi^{-1}(0)$ is smooth along the regular locus, $X_{0}$ is generically smooth, hence reduced. We now proceed by direct computation. Let us treat for example the case of $A_{r}$. Then $f(x, y)$ is quasihomogeneous of type $(2 r+2 ; 2, r+1)$, where we suppose that the weights of $x$ and $y$ are 2 and $r+1$, respectively.

Since $f$ defines a reduced curve, it must have the form $a y^{2}-b x^{r+1}$, with $a, b$ nonzero constants. After rescaling we may assume that $f$ has the form given in the statement of the proposition. The same argument works for the other cases as well. 
Now suppose that $k$ is not algebraically closed. The same argument suffices, except in the cases $A_{2 r+1}$ and $D_{2 r}$. For example, in the case $A_{2 r+1}$ one must rule out the possibility $f(x, y)=y^{2}-a x^{2 r+2}$, where $a \in k^{\times}$is a nonsquare. But the natural action map $G_{0} \times X_{0} \rightarrow \pi^{-1}(0)$ induces an injection on geometric irreducible components - see Lemma 4.14. The irreducible components of $\pi^{-1}(0)$ are geometrically irreducible, so it follows that the same must be true for $X_{0}$, hence $a$ must be a square. The same argument works for the case of type $D_{2 r}$.

At this point we have identified the central fiber of $\varphi$ with the desired curve. We will obtain the identification over the whole of $B$ via a deformation argument. Before doing this, we must determine the singularities appearing in the other fibers of $\varphi$.

Proposition 3.12. Let $t \in \mathfrak{g}_{1}$ be a semisimple element, and let $b$ denote its image in $B$. Let $D$ denote the Dynkin diagram of $Z_{G}(t)$, and write it as a disjoint union $D=D_{1} \cup \cdots \cup D_{k}$ of its connected components.

Let $y \in \varphi^{-1}(b)(k)=X_{b}(k)$ be a singular point. Then $y$ is a simple singularity of type $D_{i}$ for some $i=1, \ldots, s$.

Proof. We have an isomorphism

$$
G_{0} \times^{Z_{G_{0}}(t)}\left(t+\mathcal{N}\left(\mathfrak{z}_{\mathfrak{g}}(t)_{1}\right)\right) \cong \pi^{-1}(b),
$$

induced by the map $(g, t+n) \mapsto g \cdot(t+n)$. Let $y$ have Jordan decomposition $y=y_{s}+y_{n}$. Without loss of generality, we may suppose that $k$ is algebraically closed and that $y_{s}=t$. Then $y_{n} \in \mathfrak{z}_{\mathfrak{g}}(t)$ is a subregular nilpotent element. If we decompose $\left[\mathfrak{z}_{\mathfrak{g}}(t), \mathfrak{z}_{\mathfrak{g}}(t)\right]=\mathfrak{l}^{1} \times \cdots \times \mathfrak{l}^{k}$ into a product of simple, $\theta$-stable subalgebras then $y_{n}$ has a decomposition $y_{n}=y_{1}+\cdots+y_{k}$, where $y_{i} \in \mathfrak{l}^{i}$ is a nilpotent element. After renumbering, we can assume that $y_{1} \in \mathfrak{l}^{1}$ is a subregular nilpotent element, and all of the other $y_{i} \in \mathfrak{l}^{i}$ are regular nilpotent. Moreover, the restriction of $\theta$ to each $\mathfrak{l}^{i}$ is a stable involution.

Now fix a transverse slice $S_{1}$ to the $Z_{G_{0}}(t)$-orbit of $y_{1}$ in $\mathfrak{l}_{1}^{1}$. It then follows that $S_{1}+\sum_{j \geq 2} y_{j}$ is a transverse slice to the $Z_{G_{0}}(t)$-orbit of $y_{n}$ in $\mathcal{N}\left(\mathfrak{l}_{1}^{1}\right)$ and hence $X_{1}=t+S_{1}+\sum_{j \geq 2} y_{j}$ is a transverse slice at $y$ to the $G_{0}$ action in $\pi^{-1}(b)$, as the above isomorphism makes $\pi^{-1}(b)$ into a fiber bundle over $G_{0} / Z_{G_{0}}(t)$ with fiber $\mathcal{N}\left(\mathfrak{z}_{\mathfrak{g}}(t)_{1}\right)$.

On the other hand, we know that $X_{b}$ is also a transverse slice at $y$ to the $G_{0}$ action in $\pi^{-1}(b)$. The result now follows from Proposition 3.1 and Proposition 3.11.

Semiuniversal deformations and the proof of Theorem 3.8. We can now complete the proof of Theorem 3.8. There exists a semiuniversal deformation $\widehat{Z} \rightarrow \widehat{D}$ of the central fiber $X_{0}$ as a $\mathbb{G}_{m}$-scheme, where $\widehat{Z} \rightarrow \widehat{D}$ is a morphism of formal schemes with underlying reduced schemes given by $X_{0} \rightarrow$ Spec $k$ [Slodowy 1980b, §2.7]. 
The proof of the theorem is based on the fact that, since $X_{0}$ is given as the zero set of an explicit polynomial $f(x, y), \widehat{Z} \rightarrow \widehat{D}$ admits a canonical algebraization $Z \rightarrow D$ which we can calculate explicitly and then compare with $X \rightarrow B$.

Proposition 3.13. Let $f(x, y)$ be a polynomial in two variables, quasihomogeneous of type $\left(d ; w_{1}, w_{2}\right)$. Let $X_{0} \subset \mathbb{A}^{2}$ denote the closed subscheme defined by $f$, and suppose that $X_{0}$ has an isolated singularity at the origin. Then we can construct a semiuniversal $\mathbb{G}_{m}$-deformation of $X_{0}$ : Let $J=(\partial f / \partial x, \partial f / \partial y) \subset k[x, y]$ denote the Jacobian ideal of $f$. Then $k[x, y] / J$ is a finite-dimensional $k$-vector space, and receives an action of $\mathbb{G}_{m}$. Choose $\mathbb{G}_{m}$-invariant polynomials $g_{1}(x, y), \ldots, g_{n}(x, y)$ projecting to a $k$-basis of $\mathbb{G}_{m}$-eigenvectors of $k[x, y] / J$. Now define

$$
Z=\left\{f+t_{1} g_{1}+\cdots+t_{n} g_{n}=0\right\} \subset \mathbb{A}^{2} \times \mathbb{A}^{n},
$$

and let $\Phi: Z \rightarrow D$ denote the natural projection to the $\mathbb{A}^{n}$ factor.

Suppose that $g_{i}$ has weight $r_{i}$, and let $\mathbb{G}_{m}$ act on $t_{i}$ by the character $t \mapsto t^{d-r_{i}}$. Then $\Phi$ is a $\mathbb{G}_{m}$-equivariant morphism, and the formal completion $\widehat{\Phi}: \widehat{Z} \rightarrow \widehat{D}$ of this morphism is a semiuniversal $\mathbb{G}_{m}$-deformation of $X_{0}$.

Proof. See [Slodowy 1980b, §2.4].

Applying this to our fixed polynomial $f$, we obtain a family of curves $Z \rightarrow D$, where $D$ is an affine space of dimension $n$, and a Cartesian diagram of formal schemes:

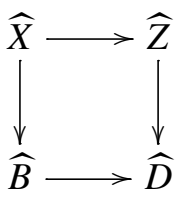

An elementary calculation shows that in each case $A_{r}, D_{r}$, or $E_{r}$, we have $n=r$ and $Z \rightarrow D$ is the family of curves appearing in the statement of Theorem 3.8. The morphism $\widehat{B} \rightarrow \widehat{D}$ is given by power series and respects the $\mathbb{G}_{m}$-actions on either side, which both have strictly positive weights; it follows that these power series are in fact polynomials, so this morphism has a canonical algebraization. We obtain a second Cartesian diagram:

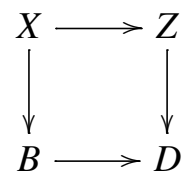

The bottom horizontal arrow is a $\mathbb{G}_{m}$-equivariant polynomial map between affine spaces of the same dimension and the weights on the domain and codomain are the same. We now apply the following lemma: 
Lemma 3.14 [Slodowy 1980b, §8.1, Lemma 3]. Let $\mathbb{G}_{m}$ act on affine spaces $V, U$ of dimension n, and let $\phi: V \rightarrow U$ be an equivariant morphism. Suppose that:

- $\mathbb{G}_{m}$ acts on $V$ and $U$ with the same strictly positive weights.

- The central fiber $\phi^{-1}(0)$ is zero dimensional.

Then $\phi$ is an isomorphism.

We must verify that the second condition holds. If $b \in B$ is mapped to $0 \in D$, then $X_{b} \cong X_{0}$. Proposition 3.12 implies that all singularities in the noncentral fibers of $\varphi$ are simple singularities belonging to simply laced root systems of rank strictly less than $r$, and so we must have $b=0$. This completes the proof of Theorem 3.8.

A lemma. The results of this section will be used later. Let $S=e+\mathfrak{z}_{\mathfrak{g}}(f)$, and let $\tau$ denote the involution of $S$ induced by $-\theta$. Thus $S$ is an affine space of dimension $r+2$, and we have $S^{\tau}=X$.

Lemma 3.15. We can choose global coordinates $z_{1}, \ldots, z_{r+2}$ on $S, u_{1}, \ldots, u_{r}$ on $B$ such that $z_{1}, \ldots, z_{r+1}$ are fixed by $\tau, \tau\left(z_{r+2}\right)=-z_{r+2}$, and such that the following holds: the morphism $X \rightarrow B$ is given by the formula

$$
\left(z_{1}, \ldots, z_{r+1}\right) \mapsto\left(z_{1}, \ldots, z_{r-1}, f\left(z_{1}, \ldots, z_{r+1}\right)\right)
$$

for some polynomial function $f$, and the morphism $S \rightarrow B$ is given by the formula

$$
\left(z_{1}, \ldots, z_{r+2}\right) \mapsto\left(z_{1}, \ldots, z_{r-1}, f\left(z_{1}, \ldots, z_{r+1}\right)+z_{r+2}^{2}\right) .
$$

Proof. We recall that there is a contracting action of $\mathbb{G}_{m}$ on $S$, and that this action sends $X$ to itself. Applying Lemma 3.9, we see that we can find $\mathbb{G}_{m}$ and $\tau$-invariant decompositions $S=V_{0} \oplus V_{1} \oplus U, B=U_{0} \oplus U$ such that the map $S \rightarrow B$ is given by $\left(v_{0}, v_{1}, u\right) \mapsto\left(\psi\left(v_{0}, v_{1}, u\right), u\right)$ for some $\mathbb{G}_{m}$-equivariant morphism $\psi$. Moreover, $\tau$ acts trivially on $V_{0} \oplus U$ and as -1 on $V_{1}$. We have $\operatorname{dim} V_{0}=2, \operatorname{dim} V_{1}=\operatorname{dim} U_{0}=1$, $\operatorname{dim} U=r-1$. Moreover, $\psi$ is quasihomogeneous of some degree.

We choose coordinates as follows: let $z_{1}, \ldots, z_{r-1}$ be arbitrary linear coordinates on $U, z_{r}, z_{r+1}$ coordinates which are eigenfunctions for the $\mathbb{G}_{m}$-action, and $z_{r+2}$ an arbitrary linear coordinate on $V_{1}$. Then [Slodowy 1980b, §7.4, Proposition 2] implies that $z_{r+2}$ has degree equal to half the degree of $\psi$. It follows that we must have $\psi\left(v_{0}, v_{1}, u\right)=\psi\left(v_{0}, 0, u\right)+z_{r+2}^{2}$, after possibly rescaling coordinates. (The coefficient of $z_{r+2}^{2}$ must be nonzero since $S_{0}$ has a unique isolated singularity.)

Corollary 3.16. Let $b \in B(k)$, and let $t \in \pi^{-1}(b)(k)$ be a semisimple element. Then there is a bijection between the connected components of the Dynkin diagram of $Z_{G}(t)$ and the singularities of the fiber $X_{b}$, which takes each (connected, simply laced) Dynkin diagram to a singularity of corresponding type. 
Proof. Lemma 3.15 implies that the singular locus of $S_{b}$ is equal to the singular locus of $X_{b}$. We have seen that the singular points of $X_{b}$ are precisely the subregular elements of $X_{b}$. It therefore suffices to show that $X_{b}$ meets each $G$-orbit of subregular elements in $p^{-1}(b)$ exactly once, or equivalently that $S_{b}$ meets each $G$-orbit of subregular elements in $p^{-1}(b)$ exactly once. This follows immediately from [Slodowy 1980b, §6.6, Proposition 2] and the remark following.

\section{Jacobians and stabilizers of regular elements}

We continue with the notation of the previous section. Thus $G$ is a split simple group of type $A_{r}, D_{r}$, or $E_{r}, \theta$ is a stable involution of $G$, and $E \in \mathfrak{g}_{1}$ is a regular nilpotent element. The pair $(\theta, E)$ is uniquely determined up to $G^{\text {ad }}(k)$-conjugacy. This data determines a regular normal $\mathfrak{s l}_{2}$-triple $(E, H, F)$. We choose further a subregular normal $\mathfrak{s l}_{2}$-triple $(e, h, f)$. Our chosen $\mathfrak{s l}_{2}$-triples give two special transverse slices: first, the Kostant section $\kappa=E+\mathfrak{z}_{\mathfrak{g}}(F)_{1}$, which is a section of the categorical quotient $\pi: \mathfrak{g}_{1} \rightarrow B$ by regular elements; second, a transverse slice to the $G_{0}$-orbit of $e, X=e+\mathfrak{z}_{\mathfrak{g}}(f)_{1}$. The fibers of the induced map $\varphi: X \rightarrow B$ are reduced connected curves.

In this section we shall write $\mathfrak{g}_{1}^{\text {rs }}$ for the open subvariety of regular semisimple elements, and $B^{\text {rs }}$ for its image in $B$. For any variety $Z \rightarrow B$ we will write $Z^{\mathrm{rs}}=Z \times{ }_{B} B^{\mathrm{rs}}$. Thus the morphism $X^{\mathrm{rs}} \rightarrow B^{\mathrm{rs}}$ is a family of smooth curves.

Homology. Fix a separable closure $K$ of $k$. In the following if $X$ is a $k$-scheme of finite type, we will write $H_{1}\left(X, \mathbb{F}_{2}\right)$ for $H_{\text {ét }}^{1}\left(X \otimes_{k} K, \mathbb{F}_{2}\right)^{*}$, the dual of the first étale cohomology of $X \otimes_{k} K$. This is a finite group, and receives an action of the Galois $\operatorname{group} \operatorname{Gal}(K / k)$.

Suppose that $A$ is a finite group scheme over $k$, killed by 2 , and that $Y \rightarrow X$ is an $A$-torsor. This defines a class in $H_{\text {ét }}^{1}\left(X \otimes_{k} K, A \otimes_{k} K\right) \cong \operatorname{Hom}\left(H_{1}\left(X, \mathbb{F}_{2}\right), A(K)\right)$. Viewing $H_{1}\left(X, \mathbb{F}_{2}\right)$ as a finite group scheme over $k$, this class defines a homomorphism $H_{1}\left(X, \mathbb{F}_{2}\right) \rightarrow A$.

Now suppose given an embedding $K \hookrightarrow \mathbb{C}$. Then there is a canonical isomorphism $H_{1}\left(X, \mathbb{F}_{2}\right) \cong H_{1}\left(X(\mathbb{C}), \mathbb{F}_{2}\right)$ with the topological homology. If $X(\mathbb{C})$ is connected and $x \in X(\mathbb{C})$, then the homomorphism $\pi_{1}(X(\mathbb{C}), x) \rightarrow A(\mathbb{C})$ induced by the torsor $Y \rightarrow X$ factors through the Hurewicz map $\pi_{1}(X(\mathbb{C}), x) \rightarrow H_{1}\left(X(\mathbb{C}), \mathbb{F}_{2}\right)$ and the induced map $H_{1}\left(X(\mathbb{C}), \mathbb{F}_{2}\right) \rightarrow A(\mathbb{C})$ agrees with the previous one, up to applying the comparison isomorphism. In particular, this map does not depend on the choice of basepoint.

If $X$ is a geometrically connected smooth projective curve over $k$, then there is a canonical isomorphism $H_{1}\left(X, \mathbb{F}_{2}\right) \cong J_{X}$ [2], where $J_{X}$ denotes the Jacobian of the curve $X$. 
Stabilizers of regular elements. Let $\mathfrak{g}_{1}^{\text {reg }} \subset \mathfrak{g}_{1}$ denote the open subset of regular elements. We write $Z \rightarrow \mathfrak{g}_{1}^{\text {reg }}$ for the stabilizer scheme, defined as the equalizer of the diagram

$$
G_{0} \times \mathfrak{g}_{1}^{\text {reg }} \underset{(g, x) \mapsto x}{\stackrel{(g, x) \mapsto g \cdot x}{\longrightarrow}} \mathfrak{g}_{1}^{\text {reg }}
$$

Proposition 4.1. 1. $Z$ is a commutative group scheme, quasifinite over $\mathfrak{g}_{1}^{\text {reg }}$.

2. $Z$ admits a canonical descent to $B$. In particular, for any two $x, y \in \mathfrak{g}_{1}^{\text {reg }}$ with the same image in $B$, there is a canonical isomorphism $Z_{G_{0}}(x) \cong Z_{G_{0}}(y)$.

Proof. The first part can be checked on geometric fibers.

For the second part, we show that $\kappa^{*} Z$ is the sought-after descent. The map $\left(G^{\mathrm{ad}}\right)^{\theta} \times \kappa \rightarrow \mathfrak{g}_{1}^{\text {reg }}$ is faithfully flat. In fact, it is étale, and [Kostant and Rallis 1971, Theorem 7] shows it to be surjective. It is now easy to construct an isomorphism between $\pi^{*} \kappa^{*} Z$ and $Z$ over this faithfully flat cover. This defines a morphism of descent data since $Z$ is commutative.

We henceforth write $Z$ for the descent to a commutative group scheme over $B$. Consider the orbit map $\mu^{\mathrm{rs}}: G_{0} \times \kappa^{\mathrm{rs}} \rightarrow \mathfrak{g}_{1}^{\mathrm{rs}}$. This map is finite and étale, and we can form the pullback square:

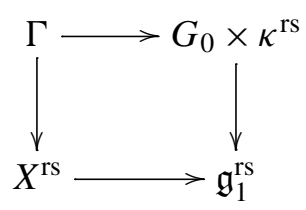

Concretely, for $b \in B^{\mathrm{rs}}(k), \Gamma_{b} \rightarrow X_{b}$ is the $Z_{b}$-torsor given by

$$
\Gamma_{b}=\left\{g \in G_{0} \mid g \cdot \kappa(b) \in X_{b}\right\} .
$$

We thus obtain a Galois-equivariant map $H_{1}\left(X_{b}, \mathbb{F}_{2}\right) \rightarrow Z_{b}$.

Theorem 4.2. If $G$ is simply connected, the map just defined is an isomorphism.

Example 4.3. Let us first illustrate the theorem in the case $G=\mathrm{SL}_{2}$. We can take $\theta$ to be conjugation by the matrix $\left(\begin{array}{ll}1 & 0 \\ 0 & -1\end{array}\right)$. Then we have

$$
\mathfrak{g}_{0}=\left\{\left(\begin{array}{cc}
a & 0 \\
0 & -a
\end{array}\right)\right\} \quad \text { and } \quad \mathfrak{g}_{1}=\left\{\left(\begin{array}{cc}
c c 0 & x \\
y & 0
\end{array}\right)\right\} .
$$

The regular nilpotents in $\mathfrak{g}_{1}$ are those with $x$ or $y$ zero but not both, and the only subregular nilpotent element in $\mathfrak{g}_{1}$ is zero. The quotient map $\mathfrak{g}_{1} \rightarrow \mathfrak{g}_{1} / / G_{0} \cong \mathbb{A}^{1}$ sends the above matrix to $x y \in \mathbb{A}^{1}$. In particular $X=\mathfrak{g}_{1}$ in this case, with the smooth fibers of the map $\varphi: X \rightarrow \mathfrak{g}_{1} / / G_{0}$ isomorphic to the punctured affine line. 
The group $G_{0}$ is isomorphic to $\mathbb{G}_{m}$, and $t \in \mathbb{G}_{m}$ acts by

$$
t \cdot\left(\begin{array}{ll}
0 & x \\
y & 0
\end{array}\right)=\left(\begin{array}{cc}
0 & t^{2} x \\
t^{-2} y & 0
\end{array}\right)
$$

The stabilizer of any regular semisimple element is $\mu_{2} \subset \mathbb{G}_{m}$, and it is clear that for any $b \in \mathbb{A}^{1}-\{0\}$, the induced map $H_{1}\left(X_{b}, \mathbb{F}_{2}\right) \rightarrow \mu_{2}$ is an isomorphism.

We now consider the proof of the theorem in the general case. It suffices to prove the theorem when $k=\mathbb{C}$, which we now assume. In what follows, we simplify notation by identifying all varieties with their complex points. Fix a choice $\mathfrak{c}$ of Cartan subspace, and let $C \subset G$ denote the corresponding maximal torus.

Now choose $x \in \mathfrak{c}$, and let $b=\pi(x) \in B$. Let $L=Z_{G}(x)$ and $\mathfrak{l}=$ Lie $L$. We write $L^{1}$ for the derived group of $Z_{G}(x)$, which is simply connected, since $G$ is. In the following, given $y \in \mathfrak{c}$, we shall write $\mathfrak{g}_{1, y}$ for the fiber of the map $\mathfrak{g}_{1} \times_{\mathfrak{c} / W} \mathfrak{c} \rightarrow \mathfrak{c}$ above $y$, and $\mathfrak{l}_{1, y}$ for the fiber of the map $\mathfrak{l}_{1} \times_{\mathfrak{c} / W(x)} \mathfrak{c} \rightarrow \mathfrak{c}$ above $y$.

Lemma 4.4. Let $y \in \mathfrak{c}^{\mathrm{rs}}$. Then there is a commutative diagram

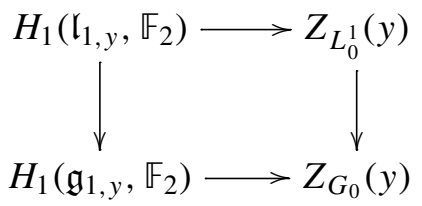

Proof. This follows from the existence of a commutative diagram

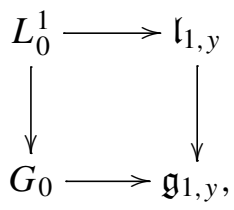

where the top row is a $Z_{L_{0}^{1}}(y)$-torsor and the bottom row is a $Z_{G_{0}}(y)$-torsor. The vertical arrows are compatible with the homomorphism $Z_{L_{0}^{1}}(y) \rightarrow Z_{G_{0}}(y)$.

Suppose that $X_{b}$ has a singular point $u=u_{s}+u_{n}$. Choose $g \in G_{0}$ such that $g \cdot u_{s}=x \in \mathfrak{c}$, and set $v=g \cdot u$. The Jordan decomposition of $v$ is $v=v_{s}+v_{n}=x+v_{n}$. Then $v_{n} \in \mathfrak{l}_{1}$ is a subregular nilpotent, corresponding to a connected component $D\left(v_{n}\right)$ of the Dynkin diagram of $L$. We choose a normal subregular $\mathfrak{s l}_{2}$-triple $\left(v_{n}, t, w\right)$ in $\mathfrak{l}$ containing $v_{n}$, and define $X^{1}=v_{n}+\mathfrak{z}_{\mathfrak{l}}(w)_{1} . X^{1}$ is a transverse slice to the $L_{0}$-orbit of $v$ in $\mathfrak{l}_{1}$, by Proposition 3.4.

Proposition 4.5. The dimension of $X^{1}$ is rank $G+1 . X^{1} \subset \mathfrak{g}_{1}$ is a transverse slice to the $G_{0}$-orbit of $v$ in $\mathfrak{g}_{1}$. 
Proof. $X^{1}$ has the correct dimension to be a transverse slice to the orbit of a subregular element, so it suffices to check the infinitesimal condition $\left[v, \mathfrak{g}_{0}\right] \cap \mathfrak{z}_{\mathfrak{l}}(w)_{1}=0$. In fact, we show that $[v, \mathfrak{g}] \cap \mathfrak{z}_{\mathfrak{r}}(w)=0$. Define

$$
V=\bigoplus_{\substack{\alpha \in \Phi_{\mathfrak{c}} \\ \alpha(x) \neq 0}} \mathfrak{g}^{\alpha} .
$$

Then $V$ is the orthogonal complement of $\mathfrak{l}$ with respect to the Killing form of $\mathfrak{g}$, and so is l-invariant. It follows that $[v, \mathfrak{g}]=[v, V] \oplus\left[v_{n}, \mathfrak{l}\right] \subset V \oplus\left[v_{n}, \mathfrak{l}\right]$. We thus have $[v, \mathfrak{g}] \cap \mathfrak{z} \mathfrak{l}(w)=\left[v_{n}, \mathfrak{l}\right] \cap \mathfrak{z} \mathfrak{l}(w)=0$.

Proposition 4.6. For all sufficiently small open neighborhoods $U$ of $u$ in $X$, there exists an open neighborhood $U_{0}$ of $b \in \mathfrak{c} / W$ such that for all $y \in \pi^{-1}\left(U_{0}\right) \cap \mathfrak{c}$ there is a commutative diagram

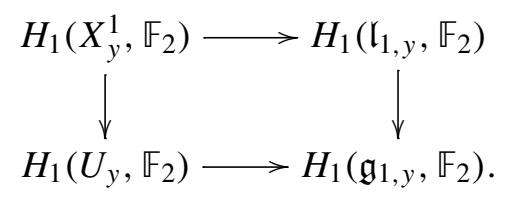

Proof. If $U$ is a sufficiently small open set around $u$ in $X$, then by Proposition 3.1 we can find an isomorphism $\psi$ between $U$ and an open neighborhood $V$ of $v$ in $X^{1}$ over $\mathfrak{c} / W$, such that $\psi(u)=v$ and the two induced maps $V \hookrightarrow \mathfrak{l}_{1} \hookrightarrow \mathfrak{g}_{1}$ and $V \cong U \hookrightarrow \mathfrak{g}_{1}$ are homotopic over $\mathfrak{c} / W$. After possibly shrinking $U$, we can assume that the image of $V$ in $\mathfrak{c} / W(x)$ maps injectively to $\mathfrak{c} / W$.

In particular, for $c$ sufficiently close to $b$ we have a commutative diagram

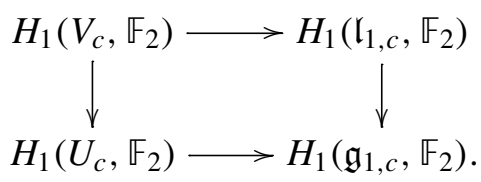

To obtain the statement in the proposition, we note that for $c$ sufficiently close to $b$ and $y \in \pi^{-1}(c) \cap \mathfrak{c}$, we can find an open subset $V_{c}^{\prime} \subset V_{c}$ such that the inclusion $V_{c}^{\prime} \subset X_{y}^{1}$ induces an isomorphism on $H_{1}$. (Use the contracting $\mathbb{G}_{m}$-action.) This completes the proof.

Corollary 4.7. With hypotheses as in Proposition 4.6, suppose in addition that $y \in \mathfrak{c}^{\mathrm{rs}}$. Let $C(x) \subset L^{1}$ be the maximal torus with Lie algebra $\mathfrak{c} \cap \mathfrak{l}^{1}$. Then there is a commutative diagram

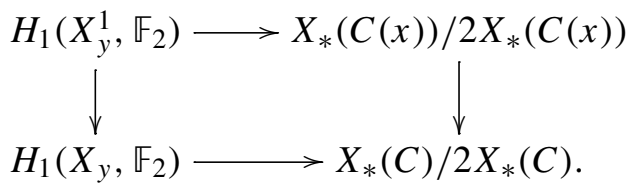


Proof. There is an isomorphism

$$
Z_{G_{0}}(y) \cong X_{*}(C) / 2 X_{*}(C),
$$

and similarly for $Z_{L_{0}^{1}}(y)$. The corollary now follows from Proposition 4.6, on noting that the map $U_{y} \rightarrow \mathfrak{g}_{1, y}$ factors through the inclusion $X_{y} \subset \mathfrak{g}_{1, y}$.

To go further, it is helpful to compare this with another description of the homology of the curves $X_{y}$.

Theorem 4.8. 1. The map $X^{\mathrm{rs}} \rightarrow \mathfrak{c}^{\mathrm{rs}} / W$ is a locally trivial fibration (in the analytic topology), and so the homology groups $H_{1}\left(X_{c}, \mathbb{F}_{2}\right)$ for $c \in B^{\mathrm{rs}}$ fit into a local system $\mathscr{H}_{1}(X)$ over $\mathfrak{c}^{\mathrm{rs}} / W$. The pullback of this local system to $\mathfrak{c}^{\mathrm{rs}}$ is constant.

2. Suppose $x \in \mathfrak{c}$ has been chosen so that $\alpha(x)=0$ for some $\alpha \in \Phi_{\mathfrak{c}}$, and the only roots vanishing on $x$ are $\pm \alpha$. Then for each $y \in \mathfrak{c}^{\mathrm{rs}}$ there is a vanishing cycle $\gamma_{\alpha} \in H_{1}\left(X_{y}, \mathbb{F}_{2}\right)$, associated to the specialization $X_{y} \rightarrow X_{x}$. This element defines a global section of the pullback of $\mathscr{H}_{1}(X)$ to $\mathrm{c}^{\mathrm{rs}}$.

3. Let $R_{\mathfrak{c}} \subset \Phi_{\mathfrak{c}}$ denote a choice of root basis. Then for each $y \in \mathfrak{c}^{\mathrm{rs}}$ the set $\left\{\gamma_{\alpha} \mid \alpha \in R_{\mathfrak{c}}\right\}$ is a basis of $H_{1}\left(X_{y}, \mathbb{F}_{2}\right)$.

It seems likely that this description of the local system $\mathscr{H}_{1}(X)$ is well-known to experts, but we have not been able to find an adequate reference in the literature. The proof of this theorem is given in Section 4 below. See in particular Lemma 4.20 for the definition of the vanishing cycle $\gamma_{\alpha}$.

Now suppose $x \in \mathfrak{c}$ has been chosen so that $\alpha(x)=0$ for some $\alpha \in \Phi_{\mathfrak{c}}$, and the only roots vanishing on $x$ are $\pm \alpha$. Then the derived group of $L$ is isomorphic to $\mathrm{SL}_{2}$. By Corollary 3.16, the fiber $X_{x}$ has a unique singularity of type $A_{1}$. For $y \in \mathfrak{c}^{\mathrm{rs}}$ sufficiently close to $x$, we have by Corollary 4.7 a diagram

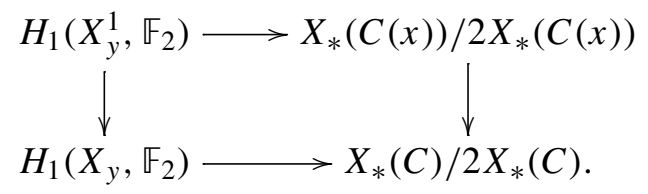

It follows from the calculations in Example 4.3 for $G=\mathrm{SL}_{2}$ that the top arrow is an isomorphism, while the right vertical arrow has image equal to the image of the set $\left\{0, \alpha^{\vee}\right\}$ in $X_{*}(C) / 2 X_{*}(C)$. Moreover, it is clear from the proof of Proposition 4.6 and the definition of the vanishing cycle (Lemma 4.20) that the image of the nontrivial element of $H_{1}\left(X_{y}^{1}, \mathbb{F}_{2}\right)$ in $H_{1}\left(X_{y}, \mathbb{F}_{2}\right)$ is exactly the vanishing cycle $\gamma_{\alpha}$. Applying the commutativity of the above diagram, we deduce that the image of $\gamma_{\alpha}$ in $X_{*}(C) / 2 X_{*}(C)$ is just $\alpha^{\vee} \bmod 2 X_{*}(C)$. Since $\gamma_{\alpha}$ comes from a global section of the local system $\mathscr{H}_{1}(X)$, we deduce the result for any $y \in \mathfrak{c}^{\mathrm{rs}}$, not just $y$ sufficiently close to $x$. 
It follows that for any $y \in \mathfrak{c}^{\mathrm{rs}}$, the map

$$
H_{1}\left(X_{y}, \mathbb{F}_{2}\right) \rightarrow Z_{G_{0}}(y) \cong X_{*}(C) / 2 X_{*}(C)
$$

takes a basis of $H_{1}\left(X_{y}, \mathbb{F}_{2}\right)$, namely the set of $\gamma_{\alpha}$ as $\alpha$ ranges over a set of simple roots, to a basis of $X_{*}(C) / 2 X_{*}(C)$, namely the corresponding set of simple coroots. This completes the proof of the theorem.

The case of $G$ adjoint. We now introduce a compactification of the family $X \rightarrow B$ of affine curves.

Lemma 4.9. The family $\varphi: X \rightarrow B$ admits a compactification to a family $Y \rightarrow B$ of projective curves. Endow $Y \backslash X$ with its reduced closed subscheme structure. Then $Y \backslash X$ is a disjoint union of smooth nonintersecting open subschemes $P_{1}, \ldots, P_{s}$, each of which maps isomorphically onto $B$. Moreover, $Y \rightarrow B$ is smooth in a Zariski neighborhood of each $P_{i}$. For each $b \in B^{\mathrm{rs}}(k), Y_{b}$ is the unique smooth projective curve containing $X_{b}$ as a dense open subset. Each irreducible component of $Y_{0}$ meets exactly one of the sections $P_{i}$.

Proof. We take the projective closure of the equations given in Theorem 3.8, and blow up any singularities at infinity. An easy calculation shows in each case that the induced family $Y \rightarrow B$ satisfies the required properties.

Let us now suppose that $G$ is adjoint, and let $G^{\mathrm{sc}} \rightarrow G$ denote its simply connected cover. We write $Z^{\text {sc }}$ for the stabilizer scheme of $G^{\text {sc }}$ over $B$. The natural map $Z^{\mathrm{sc}} \rightarrow Z$ is fiberwise surjective. Fix $b \in B^{\mathrm{rs}}(k)$. In Theorem 4.2, we saw that the inclusion $X_{b} \hookrightarrow \mathfrak{g}_{1, b}$ induces an isomorphism $H_{1}\left(X_{b}, \mathbb{F}_{2}\right) \rightarrow Z_{b}^{\text {sc }}$ of finite $k$-groups. On the other hand, we have a surjection $H_{1}\left(X_{b}, \mathbb{F}_{2}\right) \rightarrow H_{1}\left(Y_{b}, \mathbb{F}_{2}\right)$.

Theorem 4.10. The composite

$$
H_{1}\left(X_{b}, \mathbb{F}_{2}\right) \rightarrow Z_{b}^{\mathrm{sc}} \rightarrow Z_{b}
$$

factors through this surjection, and induces an isomorphism $H_{1}\left(Y_{b}, \mathbb{F}_{2}\right) \cong Z_{b}$.

By Corollary 2.12, there is a canonical alternating pairing on $Z_{b}^{\text {sc }}$, with radical equal to the kernel of the map $Z_{b}^{\mathrm{sc}} \rightarrow Z_{b}$. On the other hand, there is a pairing $(\cdot, \cdot)$ on $H_{1}\left(X_{b}, \mathbb{F}_{2}\right)$, namely the intersection product, whose radical is exactly the kernel of the map $H_{1}\left(X_{b}, \mathbb{F}_{2}\right) \rightarrow H_{1}\left(Y_{b}, \mathbb{F}_{2}\right)$. The theorem is therefore a consequence of the following result.

Theorem 4.11. The isomorphism $H_{1}\left(X_{b}, \mathbb{F}_{2}\right) \cong Z_{b}^{\text {sc }}$ preserves these alternating pairings.

Corollary 4.12. There is an isomorphism $J_{Y_{b}}[2] \cong Z_{b}$ of finite $k$-groups that takes the Weil pairing to the pairing on $Z_{b}$ defined in Corollary 2.12. 
Proof of Theorem 4.11. We can again reduce to the case $k=\mathbb{C}$. Fix a choice of Cartan subspace $\mathfrak{c}$, and let $C \subset G^{\mathrm{sc}}$ be the corresponding maximal torus. Choose $y \in \mathfrak{c}^{\mathrm{rs}}$. Let $\gamma_{\alpha} \in H_{1}\left(X_{y}, \mathbb{F}_{2}\right)$ be the element defined in Theorem 4.8. We will derive the theorem from the following statement: fix a root basis $R_{\mathfrak{c}}$ of $\Phi_{\mathfrak{c}}$, and let $\alpha, \beta \in R_{\mathfrak{c}}$ be distinct roots. Then $\left(\gamma_{\alpha}, \gamma_{\beta}\right)=1$ if $\alpha, \beta$ are adjacent in the Dynkin diagram of $\mathfrak{g}$, and $\left(\gamma_{\alpha}, \gamma_{\beta}\right)=0$ otherwise. We split the rest of the proof into two cases, according to these possibilities.

Case 1. If $\alpha, \beta$ are distinct adjacent roots, then we can choose $x \in \mathfrak{c}$ such that the elements of $\Phi_{\mathfrak{c}}$ vanishing on $x$ are exactly the linear combinations of $\alpha$ and $\beta$. Let $L=Z_{G^{\mathrm{sc}}}(x)$ and $L^{1}=L^{\mathrm{der}}$. Then $L^{1} \cong \mathrm{SL}_{3}$, and the root system $\Phi_{\mathfrak{c}}(x) \subset \Phi_{\mathfrak{c}}$ is spanned by $\alpha$ and $\beta$. Moreover, we have by Corollary 4.7 for all $y \in \mathfrak{c}^{\text {rs }}$ sufficiently close to $x$ a commutative diagram

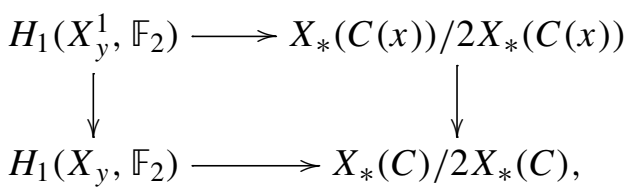

where $C(x) \subset L^{1}$ is the maximal torus with Lie algebra $\mathfrak{c} \cap \mathfrak{l}^{1}$. We know that the horizontal arrows are isomorphisms, and the vertical arrows are injective. The vertical arrows preserve the corresponding pairings.

Now, both of the objects in the top row of the above diagram are 2-dimensional $\mathbb{F}_{2}$-vector spaces, and their corresponding pairings are nondegenerate. (This is easy to see: the curve $X_{y}^{1}$ is a smooth affine curve of the form $y^{2}=x^{3}+a x+b$.) There is a unique nondegenerate alternating pairing on any 2-dimensional $\mathbb{F}_{2}$-vector space, so we deduce that $\left(\gamma_{\alpha}, \gamma_{\beta}\right)=1$.

Case 2. If $\alpha, \beta$ are distinct roots which are not adjacent in the Dynkin diagram of $\mathfrak{g}$, then we can choose $x \in \mathfrak{c}$ such that the roots vanishing on $x$ are exactly the linear combinations of $\alpha$ and $\beta$. Let $L=Z_{G}(x)$ and $L^{1}=L^{\text {der }}$. Then $L^{1} \cong \mathrm{SL}_{2} \times \mathrm{SL}_{2}$, and $X_{y}$ has exactly two singularities, each of type $A_{1}$. We can choose disjoint open neighborhoods $U_{1}, U_{2}$ of these singularities in $X$ such that for each $y \in \mathfrak{c}^{\mathrm{rs}}$ sufficiently close to $x$, the map $H_{1}\left(U_{1, y} \cup U_{2, y}, \mathbb{F}_{2}\right) \rightarrow H_{1}\left(X_{y}, \mathbb{F}_{2}\right)$ is injective and has image equal to the span of $\gamma_{\alpha}$ and $\gamma_{\beta}$. We see that these homology classes can be represented by cycles contained inside disjoint open sets of $X_{y}$. Therefore their intersection pairing is zero, and the theorem follows.

A parametrization of orbits. We suppose again that $k$ is a general field of characteristic 0 . Before stating our last main theorem, we summarize our hypotheses. We fix the following data:

- A split simple adjoint group $G$ over $k$, of type $A_{r}, D_{r}$, or $E_{r}$. 
- A stable involution $\theta$ of $G$ and a regular nilpotent element $E \in \mathfrak{g}_{1}$.

- A choice of subregular normal $\mathfrak{s l}_{2}$-triple $(e, h, f)$.

In terms of these data, we have defined:

- The categorical quotient $B=\mathfrak{g}_{1} / / G_{0}$.

- The Kostant section $\kappa \subset \mathfrak{g}_{1}$.

- A family of reduced connected curves $X \rightarrow B$.

- A family of projective curves $Y \rightarrow B$ containing $X$ as a fiberwise dense open subset.

- A stabilizer scheme $Z \rightarrow B$ whose fiber over $b \in B(k)$ is isomorphic to the stabilizer of any regular element in $\mathfrak{g}_{1, b}$.

- For each $b \in B^{\mathrm{rs}}(k)$, a natural isomorphism $J_{Y_{b}}[2] \cong Z_{b}$, that takes the Weil pairing to the nondegenerate alternating pairing on $Z_{b}$ defined in Corollary 2.12.

Proposition 4.13. For each $b \in B^{\mathrm{rs}}(k)$, there is a bijection

$$
\mathfrak{g}_{1, b}(k) / G_{0}(k) \cong \operatorname{ker}\left(H^{1}\left(k, J_{Y_{b}}[2]\right) \rightarrow H^{1}\left(k, G_{0}\right)\right),
$$

which takes the orbit of $\kappa_{b}$ to the distinguished element of $H^{1}\left(k, J_{Y_{b}}[2]\right)$.

Proof. Let $K$ be a separable closure of $k$. We recall that if $H$ is an algebraic group over $k$ which acts on a variety $X$, and $H(K)$ acts transitively on $X(K)$, then given $x \in X(k)$ there is a bijection

$$
X(k) / H(k) \cong \operatorname{ker}\left(H^{1}\left(k, Z_{H}(x)\right) \rightarrow H^{1}(k, H)\right),
$$

under which the $H(k)$-orbit of $x$ is mapped to the distinguished element, by [Gross and Bhargava 2012, Proposition 1]. We apply this with $H=G_{0}, X=\mathfrak{g}_{1, b}$, and base point $x=\kappa_{b} \in \mathfrak{g}_{1, b}(k)$ induced by the Kostant section. The result follows on using the identification $Z_{H}(x) \cong J_{Y_{b}}$ [2] of Theorem 4.10.

To go further we want to interpret the relative position of the nilpotent elements $E$ and $e$ geometrically.

Lemma 4.14. There are bijections between the following sets:

1. The set of irreducible components of $X_{0}$.

2. The set of $G_{0}$-orbits of regular nilpotent elements in $\mathfrak{g}_{1}$ containing the $G_{0}$-orbit of $e$ in their closure.

3. The set of connected components of $Y \backslash X$.

Proof. The map $\mu_{0}: G_{0} \times X_{0} \rightarrow \mathcal{N}\left(\mathfrak{g}_{1}\right)$ is flat, and so has open image. This image therefore contains all regular nilpotent $G_{0}$-orbits whose closure meets $e$. On the other hand, one checks using Proposition 2.30 that in each case the number of 
regular nilpotent $G_{0}$-orbits containing $e$ in their closure is equal to the number of irreducible components of $X_{0}$. We can therefore define a bijection between the first two sets by taking an irreducible component of $X_{0}$ to the $G_{0}$-orbit of any point on its smooth locus.

We write $Y \backslash X=P_{1} \cup \cdots \cup P_{s}$ as a disjoint union of open subschemes, each of which maps isomorphically onto $B$. By Lemma 4.9, each irreducible component of $Y_{0}$ meets a unique section $P_{i}$. We define a bijection between the first and third sets above by taking an irreducible component of $X_{0}$ to the unique section $P_{i}$ meeting its closure in $Y_{0}$.

We come now to our main theorem. We choose a section $P \cong B$ inside $Y \backslash X$, and we suppose that $E$ corresponds under the bijection of Lemma 4.14 to the unique component of $X_{0}$ whose closure in $Y_{0}$ meets $P$. For each $b \in B^{\mathrm{rs}}(k), P_{b} \in Y_{b}(k)$ defines an Abel-Jacobi map $f^{P_{b}}: Y_{b} \hookrightarrow J_{Y_{b}}$. (For the definition of this map, see [Milne 1986, §2].)

Theorem 4.15. For every $b \in B^{\mathrm{rs}}(k)$, there is a commutative diagram, functorial in $k$, and depending only on e up to $G_{0}(k)$-conjugacy:

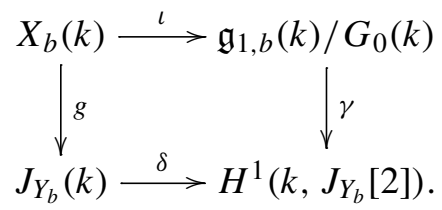

The arrows in this diagram are defined as follows:

- $\iota$ is induced by the inclusion $X_{b} \hookrightarrow \mathfrak{g}_{1, b}$.

- $g$ is the restriction of the Abel-Jacobi map $f^{P_{b}}$ to $X_{b} \subset Y_{b}$.

- $\delta$ is the usual 2-descent map in Galois cohomology associated to the exact sequence

$$
0 \rightarrow J_{Y_{b}}[2] \rightarrow J_{Y_{b}} \stackrel{[2]}{\rightarrow} J_{Y_{b}} \rightarrow 0 .
$$

- $\gamma$ is the classifying map of Proposition 4.13.

Proof. We think of the group $H^{1}\left(k, J_{Y_{b}}[2]\right)$ as classifying $J_{Y_{b}}$ [2]-torsors over $k$. With $b$ as in the theorem, let $E_{b}=[2]^{-1} f^{P_{b}}\left(Y_{b}\right) \subset J_{Y_{b}}$. We write $j_{b}: E_{b} \rightarrow Y_{b}$ for the natural map. This is a $J_{Y_{b}}$ [2]-torsor over $Y_{b}$, and the composite $\delta \circ g$ sends a point $Q \in X_{b}(k)$ to the class of the torsor $j_{b}^{-1}(Q) \subset E_{b}$.

On the other hand, we recall the $J_{Y_{b}}$ [2]-torsor $\Gamma_{b} \rightarrow X_{b}$ of (4-1), which extends uniquely to a torsor $h_{b}: D_{b} \rightarrow Y_{b}$, by Theorem 4.10. The composite $\gamma \circ \iota$ sends a point $Q \in X_{b}(k)$ to the class of $h_{b}^{-1}(Q)$. It follows from [Milne 1986, Proposition 9.1] that the two covers $D_{b} \rightarrow Y_{b}$ and $E_{b} \rightarrow Y_{b}$ become isomorphic as $J_{Y_{b}}$ [2]-torsors after extending scalars to a separable closure of $k$. To prove the theorem, it therefore 
suffices to prove that $D_{b}$ and $E_{b}$ are isomorphic as $J_{Y_{b}}$ [2]-torsors over $Y_{b}$, before extending scalars. It even suffices to prove that $h_{b}^{-1}\left(P_{b}\right)$ is always the split torsor, or in other words that $h_{b}^{-1}\left(P_{b}\right)(k)$ is not the empty set.

Let $\mu: G_{0} \times \kappa \rightarrow \mathfrak{g}_{1}$ denote the orbit map, and let $X^{\prime}$ denote the intersection of $X$ with the image of $\mu$. Because of the compatibility between $E$ and $P$, the subset $X^{\prime} \cup P$ of the underlying topological space of $Y$ is open; let $Y^{\prime}$ denote the corresponding open subscheme. Then $Y^{\prime}$ contains a Zariski open neighborhood of $P$ in $Y$.

Let $\Gamma^{\prime}=\mu^{-1}\left(X^{\prime}\right)$; this is a $Z$-torsor over $X^{\prime}$. We show that $\Gamma^{\prime}$ extends to a $Z$-torsor over $Y^{\prime}$. In fact, there is a commutative diagram with exact rows:

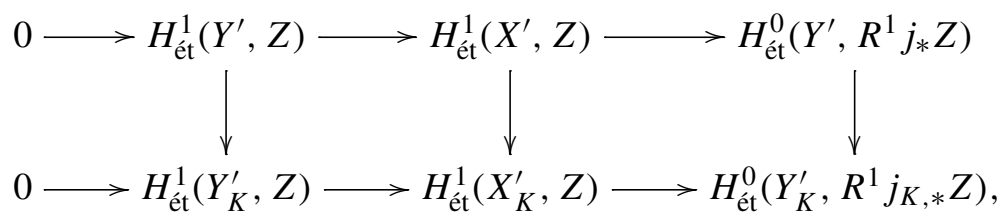

where $j: X^{\prime} \rightarrow Y^{\prime}$ is the obvious open immersion, and $(\cdot)_{K}$ denotes base change to the separable closure $K / k$. Let $i: P \hookrightarrow Y^{\prime}$ denote the complementary closed immersion. There is an isomorphism $R^{1} j_{K, *}(Z) \cong i_{K, *} Z$, and hence

$$
H_{\text {ét }}^{0}\left(Y_{K}^{\prime}, R^{1} j_{K, *}(Z)\right)=H_{\text {ét }}^{0}\left(B_{K}, Z\right) .
$$

The group $H_{\mathrm{ett}}^{0}\left(B_{K}, Z\right)$ is trivial. Indeed, the morphism $Z \rightarrow B$ is étale, while the stalk of $Z$ above the origin is trivial. The rightmost vertical arrow in the above diagram is injective, and so the class of $\Gamma^{\prime}$ in $H_{\text {ét }}^{1}\left(X^{\prime}, Z\right)$ lifts to $H_{\text {ét }}^{1}\left(Y^{\prime}, Z\right)$. We write $D^{\prime} \rightarrow Y^{\prime}$ for the corresponding torsor.

Let $F^{\prime} \rightarrow B$ denote the pullback of $D^{\prime}$ to $B \cong P \hookrightarrow Y^{\prime}$. We must show that for $b$ as in the theorem, $F_{b}^{\prime}$ is the trivial $Z$-torsor over $k$. We claim that in fact, $F^{\prime}$ is trivial. For we can choose a Zariski open neighborhood $U_{0}$ of $0 \in B$ and a Galois finite étale cover $U \rightarrow U_{0}$ such that $F^{\prime} \times{ }_{B} U$ has a trivialization as a $Z$-torsor. If $U$ is sufficiently small, then $Z(U) \hookrightarrow Z_{0}=0$ is trivial, so there is a unique such trivialization. By descent, there exists a unique trivialization of $F^{\prime}$ over $U_{0}$. The existence of the contracting $\mathbb{G}_{m}$-action on $X \rightarrow B$ now implies that $F^{\prime}$ must be globally trivial, as required. This completes the proof of the theorem.

A conjecture. We hope that the representations studied in this paper will have applications to the study of the average size of the 2-Selmer groups of the Jacobian varieties $J_{Y_{b}}$. The first step towards such applications is the following conjecture.

Conjecture 4.16. With assumptions as in Theorem 4.15, there exists a function $\eta: J_{Y_{b}}(k) \rightarrow \mathfrak{g}_{1, b}(k) / G_{0}(k)$, functorial in $k$, making the diagram 


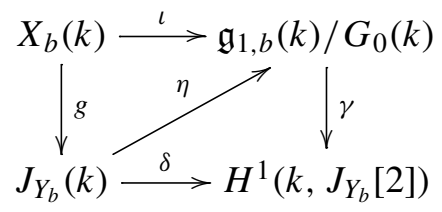

commute.

The conjecture is true in each case (namely $G$ of type $A_{2}, A_{3}$, or $D_{4}$ ) where the curves $Y_{b}$ have genus one. The representations we construct in this case are a subset of the ones studied by Bhargava and Ho in their paper on coregular representations associated to genus-one curves [Bhargava and Ho 2013], and in a forthcoming work they apply their representations to the study of the average sizes of Selmer groups [Bhargava and $\mathrm{Ho} \geq 2014$ ]. In the cases listed above one could also apply the methods of this paper, together with Bhargava's techniques for counting integral points in truncated fundamental domains, to calculate the average size of the 2Selmer groups of the curves in the corresponding families. Details will appear elsewhere.

Bhargava and Gross [2013] have shown something very close to this conjecture when $G$ is of type $A_{2 r}$. They construct rational orbits using the geometry of the intersection of two quadric hypersurfaces, and apply this to calculate the average size of the 2-Selmer groups of a certain family of hyperelliptic Jacobians. On the other hand, for some other Vinberg representations the work of Gruson, Sam and Weyman [Gruson et al. 2013] gives a relation between the geometric invariant theory and the geometry of the Jacobians of our algebraic curves, and it seems likely that this should extend to an arithmetic relation also.

The proof of Theorem 4.8. In this section we prove Theorem 4.8. Thus $G$ is a simple simply connected group over $k=\mathbb{C}, \theta$ a stable involution, and $\mathfrak{c} \subset \mathfrak{g}_{1}$ a Cartan subspace. We fix a normal subregular $\mathfrak{s l}_{2}$-triple $(e, h, f)$ in $\mathfrak{g}$, and define $S=e+\mathfrak{z}_{\mathfrak{g}}(f), X=e+\mathfrak{z}_{\mathfrak{g}}(f)_{1}=S \cap \mathfrak{g}_{1}$. Let $\tau$ denote the automorphism of $S$ induced by $-\theta$; we then have $S^{\tau}=X$. In what follows we identify all varieties with their complex points.

Lemma 4.17. Both $S^{\mathrm{rs}}$ and $X^{\mathrm{rs}}$ are locally trivial fibrations (in the analytic topology) over $\mathfrak{c}^{\mathrm{rs}} / W$.

Proof. We combine the Ehresmann fibration theorem and the existence of a good compactification for $X^{\text {rs }}$ to see that it is a locally trivial fibration over $\mathfrak{c}^{\mathrm{rs}} / W$. The corresponding result for $S$ follows from the simple relationship between $S$ and $X$, see Lemma 3.15.

Corollary 4.18. The homology groups $H_{2}\left(S_{b}, \mathbb{F}_{2}\right)$ and $H_{1}\left(X_{b}, \mathbb{F}_{2}\right)$ for $b \in \mathfrak{c}^{\mathrm{rs}} / W$ form local systems $\mathscr{H}_{2}(S)$ and $\mathcal{H}_{1}(X)$, and these local systems are canonically isomorphic. 
Proof. Only the second part needs proof. It follows either from a sheaf-theoretic argument, or from the assertion that suspension does not change the monodromy representation of a singularity, at least when one is working modulo 2; see [Arnol'd et al. 1988, Theorem 2.14].

Given $y \in \mathfrak{c}$ we write $X_{y}$ and $S_{y}$ for the respective fibers over $y$ of the maps $X \times \mathfrak{c} / W \mathfrak{c} \rightarrow \mathfrak{c}$ and $S \times \mathfrak{c} / W \mathfrak{c} \rightarrow \mathfrak{c}$.

Lemma 4.19. The local systems $\mathscr{H}_{1}(X)$ and $\mathscr{H}_{2}(S)$ become trivial after pullback to $\mathfrak{c}^{\mathrm{rs}}$.

Proof. In light of Corollary 4.18, it suffices to prove this assertion for $\mathscr{H}_{2}(S)$. The existence of the Springer resolution implies the existence of a proper morphism $\tilde{S} \rightarrow S \times \mathfrak{c} / W \mathfrak{c}$ such that for every $y \in \mathfrak{c}$, the induced map $\tilde{S} \rightarrow S_{y}$ is a minimal resolution of singularities. Moreover, $\tilde{S} \rightarrow \mathfrak{c}$ is a locally trivial fiber bundle and $\tilde{S} \times{ }_{\mathfrak{c}} \mathfrak{c}^{\mathrm{rs}} \rightarrow S \times \mathfrak{c} / W \mathfrak{c}^{\mathrm{rs}}$ is an isomorphism. See [Slodowy 1980a] for more details. These facts imply the lemma.

It follows that for any $y, z \in \mathfrak{c}^{\mathrm{rs}}$, the groups $H_{1}\left(X_{y}, \mathbb{F}_{2}\right)$ and $H_{1}\left(X_{z}, \mathbb{F}_{2}\right)$ are canonically isomorphic.

It is a consequence of Lemma 3.15 that given $b \in \mathfrak{c} / W$, a fiber $X_{b}$ has a unique nondegenerate critical point if and only if $S_{b}$ does. Let $\gamma:[0,1] \rightarrow \mathfrak{c}$ be a path such that $\gamma(t)$ is regular semisimple for $0 \leq t<1$, but such that a unique pair of roots $\pm \alpha$ vanishes on $\gamma(1)=x$. Then $X_{x}$ (or $S_{x}$ ) has a unique nondegenerate critical point, by Corollary 3.16. Let $y=\gamma(0)$. We define a homology class (that we call a vanishing cycle) $[\gamma]_{1} \in H_{1}\left(X_{y}, \mathbb{F}_{2}\right)$ as follows.

We can find local holomorphic coordinates $z_{1}, \ldots, z_{r+1}$ on $X$ centered at the critical point of $X_{b}$ and local holomorphic coordinates $u_{1}, \ldots, u_{r}$ on $\mathfrak{c} / W$ centered at $b$ such that the map $X \rightarrow \mathfrak{c} / W$ is locally of the form

$$
\left(z_{1}, \ldots, z_{r+1}\right) \mapsto\left(z_{1}, \ldots, z_{r-1}, z_{r}^{2}+z_{r+1}^{2}\right) .
$$

For $t$ close to 1, we can then define a sphere (for a suitable continuous choice of branch of $\sqrt{u_{r}(t)}$ near $\left.t=1\right)$ :

$$
S^{1}(t)=\left\{\left(u_{1}(t), \ldots, u_{r-1}(t), \sqrt{u_{r}(t)} z_{r}, \sqrt{u_{r}(t)} z_{r+1}\right) \mid z_{r}^{2}+z_{r+1}^{2}=1, \Im z_{i}=0\right\} .
$$

We define a homology class in $H_{1}\left(X_{y}, \mathbb{F}_{2}\right)$ by transporting the class of $S^{1}(t)$ for $t$ close to 1 along the image of the path $\gamma$ in $\mathfrak{c} / W$. An entirely analogous procedure defines $[\gamma]_{2} \in H_{2}\left(S_{x}, \mathbb{F}_{2}\right)$.

Lemma 4.20. The homology class of the cycle $[\gamma]_{1} \in H_{1}\left(X_{y}, \mathbb{F}_{2}\right)$ (respectively, $\left.[\gamma]_{2} \in H_{2}\left(S_{y}, \mathbb{F}_{2}\right)\right)$ is well-defined and depends only on $\alpha$. Moreover, these classes correspond under the isomorphism $H_{1}\left(X_{y}, \mathbb{F}_{2}\right) \cong H_{2}\left(S_{y}, \mathbb{F}_{2}\right)$ of Corollary 4.18. 
Proof. It is well known that the $[\gamma]_{i}$ are well-defined and depend only on the path $\gamma$ up to homotopy. It follows from Lemma 4.19 that the $[\gamma]_{i}$ depend only on the endpoint $x=\gamma(1)$ and not on the choice of path. To prove the lemma it suffices to show that $[\gamma]_{2}$ depends only on $\alpha$. In fact $[\gamma]_{2}$ is, by construction, the unique nontrivial element in the kernel of the map

$$
H_{2}\left(S_{y}, \mathbb{F}_{2}\right)=H_{2}\left(\tilde{S}_{y}, \mathbb{F}_{2}\right) \cong H_{2}\left(\tilde{S}_{x}, \mathbb{F}_{2}\right) \rightarrow H_{2}\left(S_{x}, \mathbb{F}_{2}\right) .
$$

The proof of [Shepherd-Barron 2001, Theorem 3.4] implies that there is an isomorphism of local systems $\mathscr{H}_{2}(\tilde{S}) \cong X_{*}(C) / 2 X_{*}(C)$ over $\mathfrak{c}$, and that the kernel of the map $H_{2}\left(\tilde{S}_{x}, \mathbb{F}_{2}\right) \rightarrow H_{2}\left(S_{x}, \mathbb{F}_{2}\right)$ corresponds under this isomorphism to the span in $X_{*}(C) / 2 X_{*}(C)$ of $\alpha^{\vee}$.

We can therefore define for each $\alpha \in \Phi_{\mathfrak{c}}$ a global section $\gamma_{\alpha}$ of the pullback of the local system $\mathscr{H}_{1}(X)$ to $\mathfrak{c}^{\text {rs }}$, namely the class $[\gamma]_{1}$ constructed above. Theorem 4.8 now follows from the above facts and the following result.

Lemma 4.21. Let $R_{\mathfrak{c}} \subset \Phi_{\mathfrak{c}}$ be a choice of root basis, and let $x \in \mathfrak{c}^{\mathrm{rs}}$. Then the set $\left\{\gamma_{\alpha} \mid \alpha \in R_{\mathfrak{c}}\right\}$ is a basis of $H_{1}\left(X_{x}, \mathbb{F}_{2}\right)$ as $\mathbb{F}_{2}$-vector space.

Proof. This follows immediately from the corresponding fact for the simple coroots $\left\{\alpha^{\vee} \mid \alpha \in R_{\mathfrak{c}}\right\}$.

\section{Acknowledgements}

This paper is a revised version of the author's Harvard Ph.D. thesis, written under the supervision of Benedict H. Gross. I wish to thank him for many useful suggestions and conversations.

\section{References}

[Arnol'd et al. 1988] V. I. Arnol'd, V. V. Goryunov, O. V. Lyashko, and V. A. Vasil'ev, “Особенности, I: Покальная и глобальная теория”, pp. 5-257 in Dynamical systems 6, Itogi Nauki i Tekhniki Sovrem. Probl. Mat. Fund. Napr. 6, VINITI, Moscow, 1988. Translated as "Singularities, I: Local and global theory" in Singularity theory I, Encycl. Math. Sci. 6, Springer, Berlin, 1998. MR 91h:58010b Zbl 0691.58002

[Bhargava and Gross 2013] M. Bhargava and B. H. Gross, "The average size of the 2-Selmer group of Jacobians of hyperelliptic curves having a rational Weierstrass point", preprint, 2013, available at http://www.math.harvard.edu/ gross/preprints/stable23.pdf.

[Bhargava and Ho 2013] M. Bhargava and W. Ho, "Coregular spaces and genus one curves", preprint, 2013. arXiv 1306.4424

[Bhargava and Ho $\geq 2014]$ M. Bhargava and W. Ho, "On the average sizes of Selmer groups in families of elliptic curves", In preparation.

[Bhargava and Shankar 2010] M. Bhargava and A. Shankar, "Binary quartic forms having bounded invariants, and the boundedness of the average rank of elliptic curves", preprint, 2010. To appear in Annals of Math. arXiv 1006.1002 
[Bourbaki 1968] N. Bourbaki, Groupes et algèbres de Lie, chapitres 4-6, Actualités Scientifiques et Industrielles 1337, Hermann, Paris, 1968. Translated as Lie groups and Lie algebras, Chapters 4-6, Springer, Berlin, 2008. MR 39 \#1590 Zbl 0483.22001

[Bourbaki 1975] N. Bourbaki, Groupes et algèbres de Lie, chapitres 7 et 8, Actualités Scientifiques et Industrielles 1364, Hermann, Paris, 1975. Translated in Lie groups and Lie algebras, Chapters 7-9, Springer, Berlin, 2008. Zbl 0329.17002

[Brieskorn 1971] E. Brieskorn, "Singular elements of semi-simple algebraic groups", pp. 279-284 in Actes du Congrès International des Mathématiciens (Nice, 1970), vol. 2, Gauthier-Villars, Paris, 1971. MR 55 \#10720 Zbl 0223.22012

[Collingwood and McGovern 1993] D. H. Collingwood and W. M. McGovern, Nilpotent orbits in semisimple Lie algebras, Van Nostrand Reinhold, New York, 1993. MR 94j:17001 Zbl 0972.17008

[Cook 1998] P. R. Cook, "Compactified Jacobians and curves with simple singularities", pp. 37-47 in Algebraic geometry (Catania, 1993 and Barcelona, 1994), edited by P. E. Newstead, Lecture Notes in Pure and Appl. Math. 200, Dekker, New York, 1998. MR 99j:14031 Zbl 0951.14018

[Đoković 2001] D. Ž. Đoković, "The closure diagram for nilpotent orbits of the split real form of E 7 ”, Represent. Theory 5 (2001), 284-316. MR 2002g:17019 Zbl 1050.17007

[Đoković and Litvinov 2003] D. Ž. Đoković and M. Litvinov, "The closure ordering of nilpotent orbits of the complex symmetric pair $\left(\mathrm{SO}_{p+q}, \mathrm{SO}_{p} \times \mathrm{SO}_{q}\right)$ ", Canad. J. Math. 55:6 (2003), 1155-1190. MR 2004j:22017 Zbl 1060.17002

[Esnault 1980] H. Esnault, "Sur l'identification de singularités apparaissant dans des groupes algébriques complexes", pp. 31-59 in Seminar on Singularities (Paris, 1976-1977), edited by D. T. Lê, Publ. Math. Univ. Paris VII 7, Univ. Paris VII, Paris, 1980. MR 84h:14005

[de Graaf 2011] W. A. de Graaf, "Computing representatives of nilpotent orbits of $\theta$-groups", $J$. Symbolic Comput. 46:4 (2011), 438-458. MR 2012f:17008 Zbl 1222.17002

[Gross and Bhargava 2012] B. H. Gross and M. Bhargava, "Arithmetic invariant theory", preprint, 2012, available at http://www.math.harvard.edu/ gross/preprints/invariant.pdf.

[Gruson et al. 2013] L. Gruson, S. V. Sam, and J. Weyman, "Moduli of abelian varieties, Vinberg $\theta$-groups, and free resolutions", pp. 419-469 in Commutative algebra, edited by I. Peeva, Springer, New York, 2013. Zbl 06153307 arXiv 1203.2575

[Ho 2009] W. Ho, Orbit parametrizations of curves, thesis, Princeton University, Princeton, NJ, 2009, available at http://tinyurl.com/hothesis. MR 2713823

[Humphreys 1975] J. E. Humphreys, Linear algebraic groups, Graduate Texts in Mathematics 21, Springer, New York, 1975. MR 53 \#633 Zbl 0325.20039

[Kawanaka 1987] N. Kawanaka, "Orbits and stabilizers of nilpotent elements of a graded semisimple Lie algebra”, J. Fac. Sci. Univ. Tokyo Sect. IA Math. 34:3 (1987), 573-597. MR 89j:17012 Zbl 0651.20046

[Kostant and Rallis 1971] B. Kostant and S. Rallis, "Orbits and representations associated with symmetric spaces”, Amer. J. Math. 93 (1971), 753-809. MR 47 \#399 Zbl 0224.22013

[Levy 2007] P. Levy, "Involutions of reductive Lie algebras in positive characteristic", Adv. Math. 210:2 (2007), 505-559. MR 2008b:17028 Zbl 1173.17019

[Levy 2009] P. Levy, "Vinberg's $\theta$-groups in positive characteristic and Kostant-Weierstrass slices", Transform. Groups 14:2 (2009), 417-461. MR 2010g:17022 Zbl 1209.17016

[Milne 1986] J. S. Milne, “Jacobian varieties”, pp. 167-212 in Arithmetic geometry (Storrs, CT, 1984), edited by G. Cornell and J. H. Silverman, Springer, New York, 1986. MR 861976 Zbl 0604.14018 
[Onishchik and Vinberg 1988] A. L. Onishchik and È. B. Vinberg, Семинар по группам Ли и алгебраическим группам, Nauka, Moscow, 1988. Translated as Lie groups and algebraic groups, Springer, Berlin, 1990. MR 91g:22001 Zbl 0722.22004

[Panyushev 2005] D. I. Panyushev, "On invariant theory of $\theta$-groups", J. Algebra 283:2 (2005), 655-670. MR 2006g:17022 Zbl 1071.17005

[Poonen 2013] B. Poonen, "Average rank of elliptic curves: after Manjul Bhargava and Arul Shankar", exposé no. 1049 in Séminaire Bourbaki, volume 2011/2012, Astérisque 352, Soc. Math. de France, Paris, 2013.

[Reeder 2011] M. Reeder, "Elliptic centralizers in Weyl groups and their coinvariant representations", Represent. Theory 15 (2011), 63-111. MR 2012b:20108 Zbl 1251.20042

[Reeder et al. 2012] M. Reeder, P. Levy, J.-K. Yu, and B. H. Gross, "Gradings of positive rank on simple Lie algebras”, Transform. Groups 17:4 (2012), 1123-1190. MR 3000483 Zbl 06136962

[Sekiguchi and Shimizu 1981] J. Sekiguchi and Y. Shimizu, "Simple singularities and infinitesimally symmetric spaces”, Proc. Japan Acad. Ser. A Math. Sci. 57:1 (1981), 42-46. MR 82g:17007 Zbl 0481.58009

[Shepherd-Barron 2001] N. I. Shepherd-Barron, "On simple groups and simple singularities", Israel J. Math. 123 (2001), 179-188. MR 2002c:14076 Zbl 1007.14001

[Slodowy 1980a] P. Slodowy, "Four lectures on simple groups and singularities", Communications of the Mathematical Institute 11, Rijksuniversiteit Utrecht, 1980. MR 82b:14002 Zbl 0425.22020

[Slodowy 1980b] P. Slodowy, Simple singularities and simple algebraic groups, Lecture Notes in Mathematics 815, Springer, Berlin, 1980. MR 82g:14037 Zbl 0441.14002

[Springer 1977] T. A. Springer, Invariant theory, Lecture Notes in Mathematics 585, Springer, Berlin, 1977. MR 56 \#5740 Zbl 0346.20020

[Springer 2009] T. A. Springer, Linear algebraic groups, Second ed., Birkhäuser, Boston, 2009. MR 2009i:20089 Zbl 1202.20048

[Steinberg 1974] R. Steinberg, Conjugacy classes in algebraic groups, Lecture Notes in Mathematics 366, Springer, Berlin, 1974. MR 50 \#4766 Zbl 0281.20037

[Vinberg 1976] Ѐ. В. Vinberg, “Группа Вейля градуированной алгебры Ли”, Izv. Akad. Nauk SSSR Ser. Mat. 40:3 (1976), 488-526. Translated as "The Weyl group of a graded Lie algebra" in Math. USSR-Izvestiya 10:3 (1976), 463-495. MR 55 \#3175 Zbl 0363.20035

Communicated by Bjorn Poonen

Received 2012-11-08 Revised 2013-02-14 Accepted 2013-03-17

thorne@math.harvard.edu Department of Mathematics, Harvard University,

1 Oxford Street, Cambridge, MA 02138, United States 


\section{Algebra \& Number Theory}

msp.org/ant

\section{EDITORS}

MANAGING EDITOR

Bjorn Poonen

Massachusetts Institute of Technology

Cambridge, USA

\author{
EDITORIAL BOARD CHAIR \\ David Eisenbud \\ University of California \\ Berkeley, USA
}

\section{BOARD OF EDITORS}

Georgia Benkart

Dave Benson

Richard E. Borcherds

John H. Coates

J-L. Colliot-Thélène

Brian D. Conrad

Hélène Esnault

Hubert Flenner

Edward Frenkel

Andrew Granville

Joseph Gubeladze

Roger Heath-Brown

Ehud Hrushovski

Craig Huneke

Mikhail Kapranov

Yujiro Kawamata

János Kollár

Yuri Manin

Barry Mazur

Philippe Michel
University of Wisconsin, Madison, USA

University of Aberdeen, Scotland

University of California, Berkeley, USA

University of Cambridge, UK

CNRS, Université Paris-Sud, France

University of Michigan, USA

Freie Universität Berlin, Germany

Ruhr-Universität, Germany

University of California, Berkeley, USA

Université de Montréal, Canada

San Francisco State University, USA

Oxford University, UK

Hebrew University, Israel

University of Virginia, USA

Yale University, USA

University of Tokyo, Japan

Princeton University, USA

Northwestern University, USA

Harvard University, USA

École Polytechnique Fédérale de Lausanne
Susan Montgomery

Shigefumi Mori

Raman Parimala

Jonathan Pila

Victor Reiner

Karl Rubin

Peter Sarnak

Joseph H. Silverman

Michael Singer

Vasudevan Srinivas

J. Toby Stafford

Bernd Sturmfels

Richard Taylor

Ravi Vakil

Michel van den Bergh

Marie-France Vignéras

Kei-Ichi Watanabe

Efim Zelmanov

Shou-Wu Zhang
University of Southern California, USA

RIMS, Kyoto University, Japan

Emory University, USA

University of Oxford, UK

University of Minnesota, USA

University of California, Irvine, USA

Princeton University, USA

Brown University, USA

North Carolina State University, USA

Tata Inst. of Fund. Research, India

University of Michigan, USA

University of California, Berkeley, USA

Harvard University, USA

Stanford University, USA

Hasselt University, Belgium

Université Paris VII, France

Nihon University, Japan

University of California, San Diego, USA

Princeton University, USA

PRODUCTION

production@msp.org

Silvio Levy, Scientific Editor

See inside back cover or msp.org/ant for submission instructions.

The subscription price for 2013 is US $\$ 200 /$ year for the electronic version, and $\$ 350 /$ year $(+\$ 40$, if shipping outside the US) for print and electronic. Subscriptions, requests for back issues and changes of subscribers address should be sent to MSP.

Algebra \& Number Theory (ISSN 1944-7833 electronic, 1937-0652 printed) at Mathematical Sciences Publishers, 798 Evans Hall \#3840, c/o University of California, Berkeley, CA 94720-3840 is published continuously online. Periodical rate postage paid at Berkeley, CA 94704, and additional mailing offices.

ANT peer review and production are managed by EditFLOW ${ }^{\circledR}$ from Mathematical Sciences Publishers.

\section{PUBLISHED BY}

- mathematical sciences publishers

nonprofit scientific publishing

http://msp.org/

(C) 2013 Mathematical Sciences Publishers 


\section{Algebra \& Number Theory}

Volume $7 \quad$ No. $9 \quad 2013$

Multiplicities associated to graded families of ideals Steven Dale Cutkosky

Normal coverings of linear groups

JOHN R. BRITNELL and ATTILA MARÓTI

Modularity of the concave composition generating function

George E. ANDrews, Robert C. RhOAdes and SANDER P. ZWEgERS

ANDREW NILES

Regular permutation groups of order $m p$ and Hopf Galois structures

2203

ТIMOTHY KOHL

Further evidence for conjectures in block theory

2241

BENJAMIN SAMBALE

Network parametrizations for the Grassmannian

2275

Kelli TALASKa and LAUREN Williams

Chow quotients of toric varieties as moduli of stable log maps

2313

Qile Chen and Matthew Satriano

Vinberg's representations and arithmetic invariant theory

JACK A. THORNE 